DOI https://doi.org/10.24297/jam.v20i.9066

\title{
A survey of topics related to Functional Analysis and Applied Sciences
}

\author{
Denise Huet \\ Université de Lorraine \\ INSTITUT ELIE CARTAN DE NANCY \\ B.P. 239 \\ F-54506 Vandoeuvre les Nancy Cedex \\ Denise.Huet@univ-lorraine.fr
}

\begin{abstract}
:
This survey is the result of investigations suggested by recent publications on functional analysis and applied sciences. It contains short accounts of the above theories not usually combined in a single document and completes the work of D. Huet 2017. The main topics which are dealt with involve spectrum and pseudospectra of partial differential equations, Steklov eigenproblems, harmonic Bergman spaces, rotation number and homeomorphisms of the circle, spectral flow, homogenization. Applications to different types of natural sciences such as echosystems, biology, elasticity, electromagnetisme, quantum mechanics, are also presented. It aims to be a useful tool for advanced students in mathematics and applied sciences.
\end{abstract}

\section{INTRODUCTION}

The article is divided into several sections, presented in the alphabetical order, as follows

\section{Contents}

1 Formation of patterns in reaction-diffusion systems

1.1 Linear stability analysis: stability of the trivial patterns . . . . . . . . . . . . . . . . . . . . . . . 374

1.2 Destabilization by the Türing mechanism . . . . . . . . . . . . . . . . . . . . . . . . . . . . . 375

1.3 The onset of patterns formation: the Turing/Ginsburg-Landau bifurcation . . . . . . . . . . . . . . . . 376

1.4 The particular case of the 1D-Gray-Scott model and chemistry . . . . . . . . . . . . . . . . . 376

2 Generalized Klausmeier-Gray-Scott model and echosystems

$2.1 \quad$ Initial Kausmeier model $\ldots \ldots \ldots \ldots$

2.2 Results by S. van der Stelt et al. [59] . . . . . . . . . . . . . . . . . . . . . . . . . . . . . . . 378

2.2 .1 The model $\ldots \ldots \ldots \ldots \ldots$

2.2 .2 The background states and the Turing-hopf instability . . . . . . . . . . . . . . . . . . . . . 379

$2.2 .3 \quad$ Ginzburg-Landau equation . . . . . . . . . . . . . . . . . . . . . . . . . 380

3 Gierer-Meinhardt system and biology

$3.1 \quad$ Existence and stability of a single spike solution . . . . . . . . . . . . . . . . . . . . . . . . . . 381 


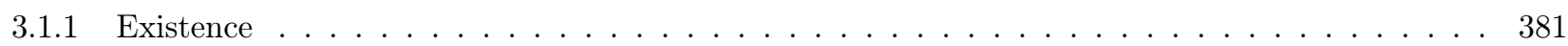

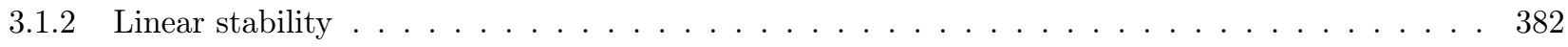

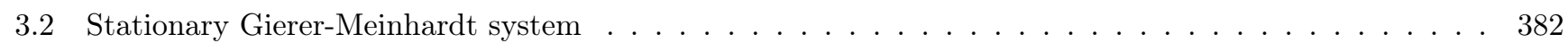

$3.3 \quad$ Existence and stability of a cluster of two spikes for the stationnary Gierer-Meinhardt system . . . . . 383

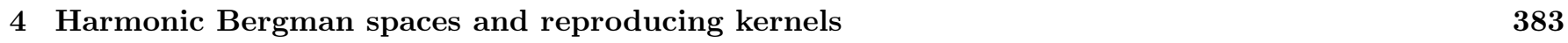

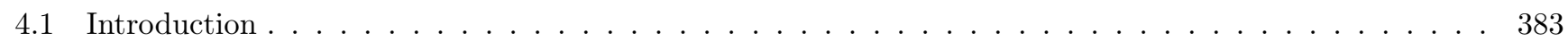

$4.1 .1 \quad$ Orthogonal decomposition of $H^{2} \cap H_{0}^{1}(\Omega) \ldots \ldots \ldots \ldots \ldots \ldots \ldots$

4.1 .2 The least positive eigenvalue of $(710 \ldots \ldots \ldots \ldots \ldots \ldots \ldots$

4.2 Subspaces of $H^{1}(\Omega)$ related to the usual Laplacian $\Delta$. Real harmonic Bergman space . . . . . . . . 385

4.2 .1 The spaces $H(\Delta, \Omega)$ and $H_{0}(\Delta, \Omega) \ldots \ldots \ldots \ldots \ldots \ldots \ldots \ldots \ldots \ldots$

4.2 .2 Orthogonal decomposition of $H(\Delta, \Omega) \ldots \ldots \ldots \ldots \ldots \ldots \ldots \ldots$

$4.2 .3 \quad$ An orthonormal basis for the real harmonic Bergman space $L_{H}^{2}(\Omega)$.

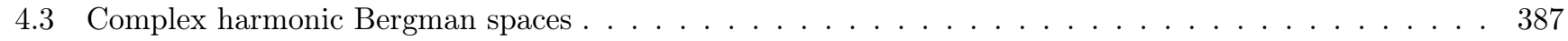

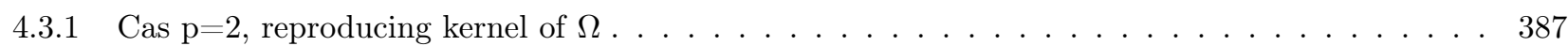

\begin{tabular}{|lll}
5 & Homeomorphisms of the circle. Rotation number & 388
\end{tabular}

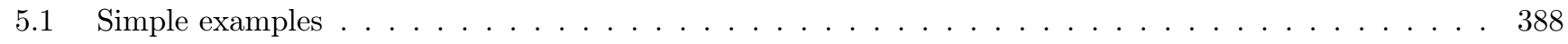

5.2 Rotation number of an orientation-preserving homeomorphism $f: S^{1} \rightarrow S^{1} \ldots \ldots \ldots 89$

$5.3 \quad$ Analytic reduction of analytic diffeomorphism of the circle to a rotation $\ldots \ldots \ldots \ldots$

5.3 .1 Arnold's Theorem . . . . . . . . . . . . . . . . . . . . . . . . . . . 390

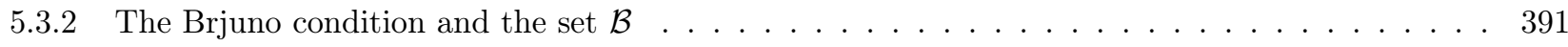

5.3 .3 The condition and the $\operatorname{set} \mathcal{H}$

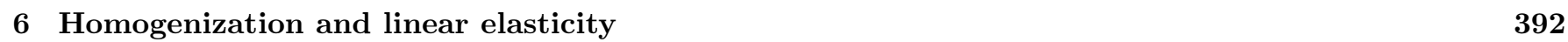

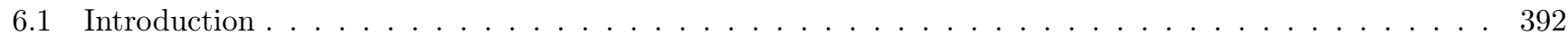

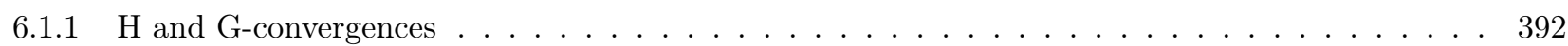

6.1 .2 Spectral problems $\ldots \ldots \ldots \ldots \ldots \ldots \ldots \ldots$

6.1 .3 Periodic homogenization $\ldots \ldots \ldots \ldots \ldots \ldots \ldots$

6.2 Homogenization and linear elasticity $\ldots \ldots \ldots \ldots \ldots \ldots \ldots$

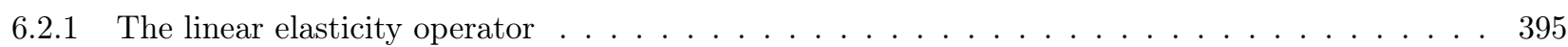


6.2 .2 Homogenization in perforated domains $\ldots \ldots \ldots \ldots \ldots \ldots \ldots$

6.3 Homogenization and Steklov eigenproblems $\ldots \ldots \ldots \ldots \ldots \ldots \ldots \ldots$

\begin{tabular}{|lll}
\hline 7 & Maxwell's equations and electromagnetisme & 397
\end{tabular}

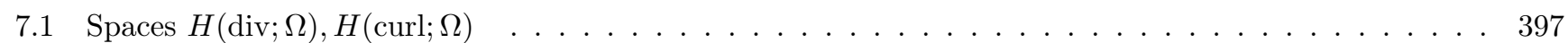

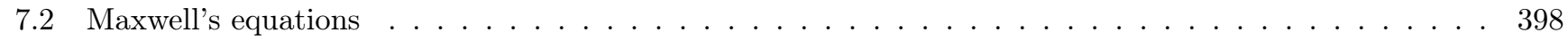

7.2 .1 Time-harmonic Maxwell's system . . . . . . . . . . . . . . . . . . . . . . . . . . . . 398

7.3 The cavity problem. $\ldots \ldots \ldots \ldots \ldots \ldots \ldots \ldots$

7.3 .1 Variational formulation $\ldots \ldots \ldots \ldots \ldots \ldots \ldots$

$7.3 .2 \quad$ Eigenvalues problem $\ldots \ldots \ldots \ldots \ldots \ldots \ldots \ldots \ldots$

7.4 The Bean model in superconductivity $\ldots \ldots \ldots \ldots$. . . . . . . . . . . . . . . 400

7.5 Bean's critical state type II superconductors $\ldots \ldots \ldots \ldots \ldots \ldots \ldots \ldots$

8 Pseudospectra and non hermitian, one dimensional, quantum mechanics 402

8.1 Introduction: closed operators $\ldots \ldots \ldots \ldots \ldots \ldots$. . . . . . . . . . . . . . . 402

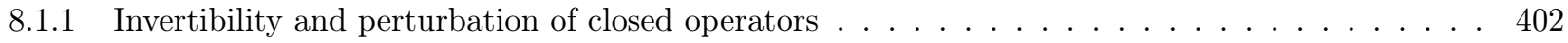

$8.1 .2 \quad$ Metrics for closed operators in a Hibert space $\ldots \ldots \ldots \ldots$. . . . . . . . . . . . . . . 402

8.2 Pseudospectra . . . . . . . . . . . . . . . . . . . . . . . . . . . . 403

$8.2 .1 \quad$ Definition of the $\epsilon$-pseudospectrum $\sigma_{\epsilon}(A) \ldots \ldots \ldots \ldots \ldots \ldots$

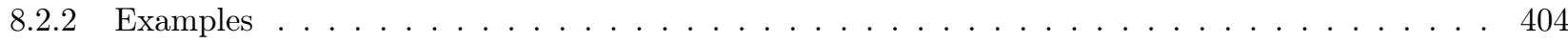

8.3 Non-hermitian one dimensional quantum mechanics $\ldots \ldots \ldots \ldots$. . . . . . . . . . . . . . 407

$8.3 .1 \quad$ Introduction to quantum mechanics $\ldots \ldots \ldots \ldots \ldots \ldots \ldots \ldots$

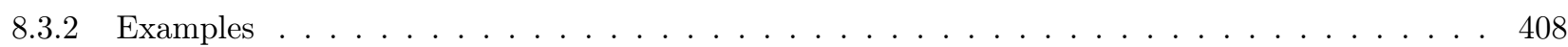

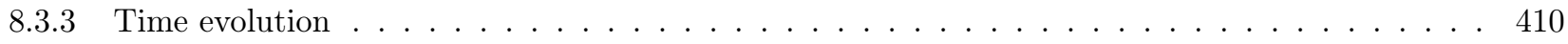

8.3 .4 Symmetrizability . . . . . . . . . . . . . . . . . . . . . . . . 410

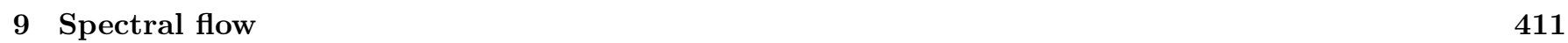

9.1 A roughly speaking description $\ldots \ldots \ldots \ldots \ldots \ldots \ldots \ldots \ldots \ldots \ldots \ldots$

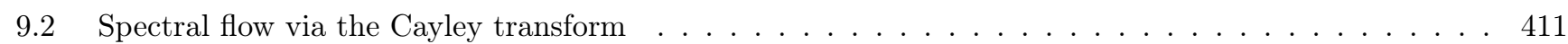

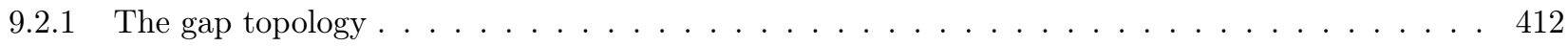

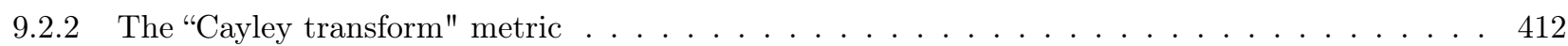




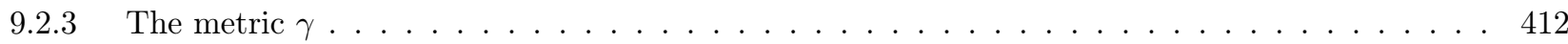

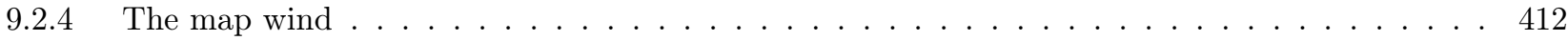

9.2 .5 Homotopy . . . . . . . . . . . . . . . . . . . . . . . . . 413

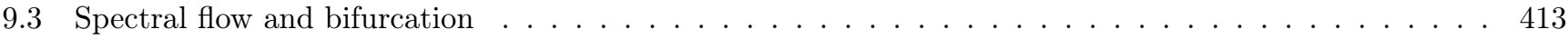

$9.3 .1 \quad$ Differentiable functions in Banach spaces . . . . . . . . . . . . . . . . . . . . . . . . . . . . 413

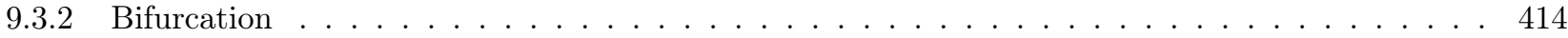

9.3 .3 Examples . . . . . . . . . . . . . . . . . . . . . . . . . . . . 414

Prepublicattions of several sections were introduced in D. Huet [34] and [35].

\section{Formation of patterns in reaction-diffusion systems}

Set-up (This section is taken from A. Doelman [20]).

Definition 1. An $N$-component reaction-diffusion system for $\mathbf{U}=\left(U_{1}, U_{2}, \ldots U_{N}\right) \in \mathbb{R}^{N}$ is a system of the form

$$
\mathbf{U}_{t}=D \Delta \mathbf{U}+\mathbf{F}(\mathbf{U}, \mu)
$$

where $\mathbf{U}(x, t)$ depends on $(x, t) \in \Omega \times \mathbb{R}^{+}$, with $\Omega \subset \mathbb{R}^{n}, D$ is a diffusion matrix i.e. a diagonal $N \times N$ matrix with strictly positives entries, $\Delta$ is the Laplace/diffusion operator, $\mu \in \mathbb{R}^{m}$ represents parameters and the vector field $\mathbf{F}(\mathbf{U}, \mu): \mathbb{R}^{N} \rightarrow \mathbb{R}^{N}$ represents the nonlinear reaction terms.

Definition 2. In [58], A.M. Turing wrote "a system of chemical substances, called morphogens, reacting together and difffusing through a tissue, is adequate to account for the main phenomena of morphogenesis. Such a system, although it may originally be quite homogeneous, may later develop a pattern or structure due to an instability of the homogeneous equilibrium.

Definition 3. The trivial patterns of (1) are solutions $\overline{\mathbf{U}}=\left(\bar{U}_{1}, \ldots \bar{U}_{N}\right)$ of the algebraic equations:

$$
\mathbf{F}(\overline{\mathbf{U}}, \mu)=0 \text { or } \quad F_{1}(\overline{\mathbf{U}}, \mu)=0, \ldots, F_{N}(\overline{\mathbf{U}}, \mu)=0
$$

From now on, $\mathrm{N}=2, \mathrm{n}=1, \Omega=\mathbb{R}\left(\mathrm{Cf}\right.$. 20], Section 2). Following the notations of [20], set $\bar{U}_{1}=\bar{U}, \overline{U_{2}}=\bar{V}, F_{1}=G, F_{2}=$ $H$.

\subsection{Linear stability analysis: stability of the trivial patterns}

Linearizing (1) around the trivial pattern $\left(\bar{U}+\alpha e^{i k x+\lambda t}, \bar{V}+\beta e^{i k x+\lambda t}\right)$, we obtain the $2 \times 2$ linear eigenvalue problem:

$$
\mathcal{A}(k, \mu)\left(\begin{array}{l}
\alpha \\
\beta
\end{array}\right)=\left(\begin{array}{cc}
g_{u}-k^{2} & g_{v} \\
h_{u} & h_{v}-d k^{2}
\end{array}\right)\left(\begin{array}{l}
\alpha \\
\beta
\end{array}\right)=\lambda\left(\begin{array}{l}
\alpha \\
\beta
\end{array}\right)
$$


where

and where

$$
g_{u}(\mu)=\frac{\partial G}{\partial U}(\bar{U}(\mu), \bar{V}(\mu) ; \mu), \quad g_{v}(\mu)=\frac{\partial G}{\partial V}(\bar{U}(\mu), \bar{V}(\mu) ; \mu),
$$

$$
h_{u}(\mu)=\frac{\partial H}{\partial U}(\bar{U}(\mu), \bar{V}(\mu) ; \mu), \quad h_{v}(\mu)=\frac{\partial H}{\partial V}(\bar{U}(\mu), \bar{V}(\mu) ; \mu),
$$

The charasteristic polynomial associated to 3 is

$$
\lambda^{2}-\left[\left(g_{u}+h_{v}\right)-(1+d) k^{2}\right] \lambda+\left[\left(g_{u}-k^{2}\right)\left(h_{v}-d k^{2}\right)-g_{v} h_{u}\right]=0
$$

It defines 2 functions $k \in \mathbb{R} \rightarrow \lambda_{1,2}(k ; \mu) \in \mathbb{C}$ such that (for instance) $\operatorname{Re}\left(\lambda_{2}(k)\right) \leq \operatorname{Re}\left(\lambda_{1}(k)\right)$. The trivial state $(\bar{U}, \bar{V})$ is spectrally stable for the values of $\mu$, for which

$$
\operatorname{Re}\left(\lambda_{2}(k ; \mu)\right) \leq \operatorname{Re}\left(\lambda_{1}(k ; \mu)\right)<0,
$$

for all $k \in \mathbb{R}$.

\subsection{Destabilization by the Türing mechanism}

As $k \rightarrow \infty, \lambda_{1} \sim-d k^{2}$ and $\lambda_{2} \sim-k^{2}$ (or vice versa), and condition (7) is satisfied. Let $\mu_{c}$ be the critical value beyond which there are values of $k$ for which $\operatorname{Re}\left(\lambda_{1}(k, \mu)\right)>0$, and $k_{c}$ such that

$$
\operatorname{Re}\left(\lambda_{1}\left( \pm k_{c} ; \mu_{c}\right)=0 \text { and } \operatorname{Re}\left(\lambda_{2}\left(k, \mu_{c}\right)\right)>0, \forall k \in \mathbb{R}\right.
$$

The planar reaction ODE, associated to (1), with $(U(x, t), V(x, t))=(u(t), v(t))$ is considered:

$$
\dot{u}=G(u, v ; \mu), \quad \dot{v}=H(u, v ; \mu) .
$$

The following notations are introduced when $\mu$ is taken just beyond $\mu_{c}$, and $\epsilon$ is smalll

$$
\mu=\mu_{c}+\epsilon^{2} \tilde{\mu}, \quad k=k_{c}+\epsilon \tilde{k}
$$

and

$$
\lambda_{1}(k, \mu)=\epsilon^{2} \tilde{\lambda}(\tilde{k}, \tilde{\mu}), \quad \lambda_{\mu}^{c}=\frac{\partial \lambda_{1}}{\partial \mu}\left(k_{c}, \mu_{c}\right) \quad \lambda_{k^{2}}^{c}=\frac{\partial^{2} \lambda_{1}}{\partial k^{2}}\left(k_{c}, \mu_{c}\right), \quad a(c, \tilde{\mu}, k)=\sqrt{\frac{2 \lambda_{\mu}^{c} \tilde{\mu}}{\left|\lambda_{k^{2}}^{c}\right|}}
$$

Under the conditions

$$
g_{u}<0, h_{v}>0, g_{v} h_{u}<0,0<d<1 \text { or } g_{u}>0, h_{v}<0, g_{v} h_{u}>0, d>1,
$$

it is proved $\left(\left[20\right.\right.$, Lemma 2.1) that the trivial state $(\bar{U}, \bar{V})$ loses stability as $\tilde{\mu}$ crosses through 0 . For $\tilde{\mu} \lambda_{\mu}^{c}>0$, there are two symmetric intervals $k= \pm k_{c}+\epsilon \tilde{k}$ with $\tilde{k} \in(-a(c, \tilde{\mu}, k)+O(\epsilon), a(c, \tilde{\mu}, k)+O(\epsilon))$, such that there exist (real) perturbations of $(\bar{U}, \bar{V})$ of the form

$$
e^{i\left(k_{c}+\epsilon \tilde{k}\right) x+\epsilon^{2} \tilde{\lambda}(\tilde{k}, \tilde{\mu}) t}\left(\begin{array}{c}
\alpha_{1}^{c}+O(\epsilon) \\
\beta_{1}^{c}+O(\epsilon)
\end{array}\right)+c . c .
$$

where $\left(\alpha_{1}^{c}, \beta_{1}^{c}\right)$ are the eigenvectors of $\mathcal{A}\left(k_{c}, \mu_{c}\right)$ corresponding to the eigenvalue $\lambda_{1}\left(k_{c}, \mu_{c}\right)=0$. With

$$
\xi=\epsilon x, \tau=\epsilon^{2} t, E_{c}(x)=e^{i k_{c} x},
$$

the above result can be written

$$
\left(\begin{array}{l}
U(x, t) \\
V(x, t)
\end{array}\right)=\left(\begin{array}{l}
\bar{U} \\
\bar{V}
\end{array}\right)+\epsilon A(\xi, \tau)\left(\begin{array}{l}
\alpha_{1}^{c} \\
\beta_{1}^{c}
\end{array}\right) E_{c}(x)+c . c .+O\left(\epsilon^{2}\right)
$$


where $A(\xi, \tau): \mathbb{R} \times \mathbb{R}^{+} \rightarrow \mathbb{C}$ satisfies the Ginzburg-Landau equation

$$
A_{\tau}=-\frac{1}{2} \lambda_{k^{2}}^{c} A_{\xi \xi}+\tilde{\mu} \lambda_{\mu}^{c} A+L A|A|^{2}
$$

Here, $L \in \mathbb{R}$ is the Landau coefficient.

\subsection{The onset of patterns formation: the Turing/Ginsburg-Landau bifurcation}

It is assumed that $L<0$. In fact, the author ([20]) shows that no (small amplitude) Turing patterns can exists beyond the destabilization if $L>0$. Introducing the new variables

$$
\hat{\tau}=\tilde{\mu} \lambda_{\mu}^{c} \tau, \quad \hat{\xi}=a(c, \tilde{\mu}, k) \xi, \quad \hat{A}=\sqrt{\frac{|L|}{\tilde{\mu} \lambda_{\mu}^{c}}} A
$$

and dropping the hats, equation 16 becomes

$$
A_{\tau}=A_{\xi \xi}+A-A|A|^{2}
$$

This equation has a family of stationary spatially periodic solutions $A(\xi, \tau)=R e^{i K \xi)}, R>0$ with

$$
K^{2}+R^{2}=1 \text { and } \quad-1<K<1
$$

and by the Eckhaus/Benjamin-Feir-Newell criterion (cf. subsubsection 2.2.3), this periodic solution is spectrally stable for $-\frac{1}{\sqrt{3}}<K<\frac{1}{\sqrt{3}}$ and unstable for $|K| \in\left(\frac{1}{\sqrt{3}}, 1\right)([20]$, Lemma 2.3).

The main result. Under the above conditions, when $\epsilon$ is sufficiently small, a Turing/Ginzburg-Landau bifurcation takes place as $\tilde{\mu}$ crosses through 0 i.e. for $\tilde{\mu} \lambda_{\mu}^{c}>0$, there exists a continuous band of asymptotically stable stationary spatially periodic patterns $\left(U_{p}(x ; k), V_{p}(x ; k)\right)$ of $(1)$, with wave number

$$
k(K)=k_{c}+\epsilon a(c, \tilde{\mu}, k) K
$$

and $-\frac{1}{\sqrt{3}}+O(\epsilon)<K<\frac{1}{\sqrt{3}}+O(\epsilon)$. These spatially periodic patterns are $O(\epsilon)$ close to the trivial state $(\bar{U}, \bar{V})$ and are approximated by

$$
\left(\begin{array}{c}
U_{p}(x ; k, \theta) \\
V_{p}(x ; k, \theta
\end{array}\right)=\left(\begin{array}{c}
\bar{U} \\
\bar{V}
\end{array}\right)+\epsilon\left(\sqrt{1-K^{2}}+O(\epsilon)\right) a(c, \tilde{\mu}, k)\left(\begin{array}{c}
\alpha_{1}^{c} \cos (k(K) x+\theta)+O(\epsilon) \\
\beta_{1}^{c} \cos (k(K) x+\theta)+O(\epsilon)
\end{array}\right)
$$

for any phase shift $\theta \in \mathbb{R},[20$, Theorem 2.4 .

Remark 1. In [20], Section 2.5, the author investigates the case of Hopf bifurcation in (9) i.e. the generation of patterns as $\mu$ passes through the critical value $\mu_{c}$.

\subsection{The particular case of the 1D-Gray-Scott model and chemistry}

(Cf. David S. Morgan et al. [42]). The Gray-Scott model, related to chemical rections between two species $\mathcal{U}$ and $\mathcal{V}$, consists of the reaction-diffusion system

$$
\begin{aligned}
& U_{t}=D_{U} \Delta U-U V^{2}+A(1-U) \\
& V_{t}=D_{V} \Delta V+U V^{2}-B V
\end{aligned}
$$


Where $U=U(x, t), x \in \mathbb{R}, V=V(x, t)$, are the concentrations of $\mathcal{U}$ (inhibitor) and $\mathcal{V}$ (activtator), $A$ and $B$ are rate constants, $D_{U}$ and $D_{V}$ are diffusivities. Here, for convenience, $D_{U}=1$ and $D_{V}=\delta^{2 \sigma}, 0<\delta \leqslant 1, \sigma>0$.The stationary states (trivial patterns) solutions of the system

$$
-U V^{2}+A(1-U)=0 \text { and } \quad U V^{2}-B V=0
$$

are $U \equiv 1, V \equiv 0$ and, when $4 B^{2}<A$,

$$
\left(U_{ \pm}, V_{ \pm}\right)=\left(\frac{1}{2}\left[1 \pm \sqrt{1-\frac{4 B^{2}}{A}}\right], \frac{A}{2 B}\left[1 \mp \sqrt{1-\frac{4 B^{2}}{A}}\right]\right)
$$

The state $(U \equiv 1, V \equiv 0)$ is is linearly stable for all $A, B>0$. On the other hand, it is proved ([42], p.117) that the state $\left(U_{+}, V_{+}\right)$cannot be marginally stable (cf. Definition 6) Therefore the authors focus only on $\left(U_{-}, V_{-}\right)$.

Linear stability of $\left(U_{-}, V_{-}\right)$, Turing/Ginsburg-Landau bifurcation. Linearizing 22 around the stationary state $\left(U_{-}, V_{-}\right)$leads to

$$
\left(\begin{array}{c}
\dot{U} \\
\dot{V}
\end{array}\right)=M\left(\begin{array}{l}
U \\
V
\end{array}\right)
$$

where

$$
M=\left(\begin{array}{cc}
-k^{2}-V_{-}^{2}-\delta^{\alpha} a & -2 \delta^{\beta} b \\
V_{-}^{2} & -\delta^{2 \sigma} k^{2}+\delta^{\beta} b
\end{array}\right)
$$

with $A, B$ rescaled as $A=\delta^{\alpha} a, B=\delta^{\beta} b, \alpha, \beta \geq 0$. The analysis of the eigenvalues of $M$ shows that $\left(U_{-}, V_{-}\right)$is linearly stable if and only if $2 \alpha \leq 3 \beta$ and determines the values $a_{c}$ and $k_{c}$ of the parameter $a$ and the wavenumber $k$ such that $\left(U_{-}, V_{-}\right)$is marginally stable. In particular,

$$
a_{c}^{2}=(3-2 \sqrt{2}) b^{3}
$$

Remark 2. 1) With the above scaling for $A$ and $B$, one has, to leading order

$$
\left(U_{-}, V_{-}\right)=\left(\delta^{2 \beta-\alpha} \frac{b^{2}}{a}, \delta^{\alpha-\beta} \frac{a}{b}\right)
$$

2) For the Gray-Scott model, the Ginsburg-Landau equation (cf. (16) ) has the form

$$
\mathcal{A}_{\tau}=\frac{2}{\sqrt{b}} \mathcal{A}+2 \sqrt{2} \mathcal{A}_{\xi \xi}-\frac{2}{9}(10 \sqrt{2}-7)|\mathcal{A}|^{2} \mathcal{A}
$$

After setting $\mathcal{A}(\xi, \tau)=R e^{i \kappa \xi}$, solutions $R$ and $\kappa$ satisfy the equation

$$
\kappa^{2}+\frac{1}{18}(20-7 \sqrt{2}) R^{2}=\frac{1}{\sqrt{2 b}}
$$

The main result. Finally, the following result is obtained ([42], Theorem 3.2): Let $a=a_{c}-\gamma^{2}$ and $3 \beta=2(\sigma+\alpha)$. For $0<\gamma \ll 1$ small enough, there exists a one parameter family of stationary spatially-periodic solutions of $[22)$ (cf definition 4 that are close to the stationary state $\left(U_{-}, V_{-}\right)$:

$$
\left(\begin{array}{l}
U(x, \kappa) \\
V(x, \kappa)
\end{array}\right)=\left(\begin{array}{l}
U_{-} \\
V_{-}
\end{array}\right)+\gamma R e^{i\left(k_{c}+\gamma \kappa\right) \delta^{\beta-\alpha} x}\left(\begin{array}{c}
2 \delta^{2 \beta-\alpha} \\
-(2-\sqrt{2}) \delta^{\alpha-\beta}
\end{array}\right)+\text { c.c. }+ \text { h.o.t. }
$$

where $R$ and $\kappa$ are related by $(30)$. 


\section{Generalized Klausmeier-Gray-Scott model and echosystems}

\subsection{Initial Kausmeier model}

In [37, C. A. Klausmeier considers the nondimensionalized system:

$$
\begin{aligned}
& \frac{\partial w}{\partial t}=a-w-w n^{2}+\nu \frac{\partial w}{\partial x} \\
& \frac{\partial n}{\partial t}=w n^{2}-m n+\left(\frac{\partial^{2}}{\partial x^{2}}+\frac{\partial^{2}}{\partial y^{2}}\right) n
\end{aligned}
$$

for water $w$ and plant biomass $n$, defined on an infinite two-dimensional domain indexed by $x$ and $y$. In $(32), a$ controls water input, $m$ measures plant losses and $\nu$ contols the rate at which water flows downhill. The corresponding nonspatial model is

$$
\begin{aligned}
& a-w-w n^{2}=0 \\
& w n^{2}-m n=0
\end{aligned}
$$

He shows that this model has a bare stable state $\hat{w}=a, \hat{n}=0$ and an other one vegetated. These stable states correspond to spatially homogeneous equilibria of $(32)$. Then he uses linear stabiliy analysis, for system 32 , in two cases: hillsides i.e. $\nu \gg 0$, and flat ground. On hillsides, for given $m$ and $\nu$, there is a critical value of water input $a$, below which Êregular stripes form. Moreover, this pattern oscillates in time and the strip moves uphill because the eigenvalue that determines the instability of this homogeneous equilibrium is complex with negative imaginary part. On flat ground, regular pattern formation is impossible when the spatially homogeneous equilibrium is stable. Numerical solutions of $(32)$ show that, in this case, irregular patterns can arise but for parameters which are ecologically unrealistic. His results are illustrated by figures obtained with ecologically realistic parameters.

\subsection{Results by S. van der Stelt et al. [59]}

\subsubsection{The model}

On one-dimensional domain, the model introduced by C. A. Klausmeier reads:

$$
\begin{aligned}
& u_{t}=k_{o} u_{x}+k_{1}-k_{2} u-k_{3} k_{5} u v^{2} \\
& v_{t}=d_{v} v_{x x}-k_{4} v+k_{5} u v^{2}
\end{aligned}
$$

where $u(x, t), v(x, t): \mathbb{R} \times \mathbb{R}_{+} \rightarrow \mathbb{R}$, and $k_{i} \geq 0, i=0, \ldots, 5, d \geq 0$. The flow of water is denoted by $u_{t}$, the slope of the aera by $k_{o} u_{x}$, the constant precipitation rate by $k_{1}$, an evaporation rate by $-k_{2} u$, and an infiltration rate by $-k_{3} k_{5} u v^{2}$. The change of biomass is assumed to be controlled by a diffusion term $d_{v} v_{x x}$. The death rate is denoted by $-k_{4} v$ and the infiltration feedback by $k_{5} u v^{2}$. In [59], system (34) is completed by the nonlinear diffusion term $d_{u}\left(u^{\gamma}\right)_{x x}$ :

$$
\begin{aligned}
& u_{t}=d_{u}\left(u^{\gamma}\right)_{x x}+k_{o} u_{x}+k_{1}-k_{2} u-k_{3} k_{5} u v^{2} \\
& v_{t}=d_{v} v_{x x}-k_{4} v+k_{5} u v^{2}
\end{aligned}
$$

where $\gamma \geq 1$ and $0<d_{v} \ll d_{u}$, and rescaled as

$$
\begin{aligned}
& U_{t}=U_{x x}^{\gamma}+C U_{x}+A(1-U)-U V^{2} \\
& V_{t}=\delta^{2 \sigma} V_{x x}-B V+U V^{2}
\end{aligned}
$$

with $0<\delta \ll 1$ and

$$
\delta^{2 \sigma}=\frac{d_{v}}{d_{u}}\left(\frac{k_{2}}{k_{1}}\right)^{\gamma-1}, \quad \sigma>0 .
$$


Remark 3. For ecosystems without a slope $k_{0}=0$ and therefore

$$
C=k_{0} \frac{k_{2}^{2} k_{3}}{k_{1}^{2} k_{5}}\left[d_{u} \frac{k_{3}}{k_{5}}\left(\frac{k_{1}}{k_{2}}\right)^{\gamma-3}\right]^{-\frac{1}{2}}=0
$$

Definition 4. Spatially periodic solutions (patterns) or wave trains are solutions $u(x, t)$ that can be written

$$
u(x, t)=u_{p}(\kappa x+\Omega t)
$$

that satisfy $u_{p}(\xi)=u_{p}(\xi+2 \pi)$. Here $\kappa$ is called the nonlinear wave number.

Definition 5. A Busse balloon for system $(36)$ is a set $\mathcal{B}$ in the $(A, \kappa)$-space with the following property: a point $(A, \kappa)$ lies in $\mathcal{B}$ if system 36 with parameter $A$ allow for at least one stable periodic solution $\left(U_{p}, V_{p}\right)$ with parameter $\kappa(\operatorname{cf} .([59, \mathrm{p} .66)$.

It is pointed out that the ecologically relevant parameter values of $\gamma$ are $\gamma=1$ or $\gamma=2$. For $\gamma=1$ and $C=0$, system (36] is the gray-Scott system 22]. In [42] Section 6.2, the existence of a Busse balloon, for the Gray-Scott model, is investigated.

\subsubsection{The background states and the Turing-hopf instability}

The model (36) has the same homogeneous background states as the Gray-Scott model for $A>4 B^{2}$, namely (cf 23 and (24)).

$$
U_{0}=1, V_{0}=0, \text { and } \quad\left(U_{ \pm}, V_{ \pm}\right)=\left(\frac{1}{2 A}\left[A \mp \sqrt{A^{2}-4 A B^{2}}\right), \frac{1}{2 B}\left(A \pm \sqrt{A^{2}-4 A B^{2}}\right) .\right.
$$

The state $\left(U_{0}, V_{0}\right)=(1,0)$ represents the desert since, in this case, $v=\frac{k_{1}}{k_{2} k_{3}} V_{0}=0$. The state $\left(U_{-}, V_{-}\right)$does not represent a homogeneously vegetated state. By linearization of (36) about the state $u_{+}=\left(U_{+}, V_{+}\right)$we have :

$$
u_{t}=\mathcal{D} u_{x x}+\mathcal{C} u_{x}+\partial_{u} F\left(u_{+} ; A, B\right) u=: \mathcal{L}\left(\partial_{x}\right) u
$$

with $u=(U, V), F(U, V ; A, B)=\left(A(1-U)-U V^{2},-B V+U V^{2}\right)$, and suitable matrices $\mathcal{C}$ and $\mathcal{D}$. Let $\mathcal{M}$ be the matrix defined by

$$
\mathcal{M}(a, c, i k)=\left(\begin{array}{cc}
-\gamma\left(U_{+}\right)^{\gamma-1} k^{2}+i c \delta^{\nu} k-V_{+}^{2}-\delta^{\alpha} a & -2 b \delta^{\beta} \\
V_{+}^{2} & -\delta^{2 \sigma} k^{2}+\delta^{\beta} b
\end{array}\right)=\mathcal{L}(i k)
$$

where $0<\delta \ll 1$ and

$$
A=a \delta^{\alpha}, \quad B=b \delta^{\beta}, \quad C=c \delta^{\nu} ; \quad \alpha, \beta>0, \quad \nu \in \mathbb{R},
$$

with $a, b, c,=O(1)$ with respect to $\delta$. Here $k$ is refered to as the linear wavenumber.

Remark 4. For ecosystems without a slope, $\mathrm{C}=0$ (cf. remark 3 ) and therefore $c=0$.

Remark 5. With the above scaling, $\left(U_{+}, V_{+}\right)$can be written out to leading order in $\delta$

$$
\left(U_{+}, V_{+}\right)=\left(\frac{b^{2}}{a} \delta^{2 \beta-\alpha}, \frac{a}{b} \delta^{\alpha-\beta}\right)+\text { h.o.t. }
$$

The $L^{2}$-spectrum of 41 is the set of $\lambda \in \mathbb{C}$ such that

$$
d(\lambda, i k)=\operatorname{det}[\mathcal{M}(a, c, i k])-\lambda]=0
$$


Definition 6. $\mathcal{L}\left(\partial_{x}\right)$ is called marginally stable with critical Fourier mode $u_{0} e^{i k_{*} x}$ associated to the critical eigenmode $i \omega_{*}$, up to complex conjugaison, if

$$
d\left(i \omega_{*}, i k_{*}\right)=0, d\left(i \omega_{*}, i k\right) \neq 0 \text { for all } k \neq \pm k_{*}
$$

and $d(\lambda, i k) \neq 0$, for all $k \in \mathbb{R}$, all $\lambda \in \mathbb{C}$ with $\lambda \neq i \omega_{*}$ and $\operatorname{Re} \lambda \geq 0$. In this case, we also say that the background state $\left(U_{+}, V_{+}\right)$of 36 is marginally stable.

Definition 7. The instability is called a Turing-Hopf [resp. Turing] instability if $k_{*} \neq 0, \omega_{*} \neq 0\left[\mathrm{resp} . k_{*} \neq 0, \omega_{*}=\right.$ $0]$.

Main results:1) when $\mathbf{C}=\mathbf{0},\left(U_{+}, V_{+}\right)$is marginally stable for $\sigma, a=a_{*}, k=k_{*}$ satisfying, to leading order in $\delta$,

$$
(2 \gamma+1) \beta-(\gamma+1) \alpha=2 \sigma, k_{*}^{2}=\frac{1}{2}(1-g) b \delta^{-2 \gamma \beta+(\gamma+1) \alpha}, a_{*}^{\gamma+1}=g \gamma b^{2 \gamma+1} .
$$

where $g=3-2 \sqrt{2},([59]$, Proposition 1),

2) when $\mathbf{C}=c \delta^{\frac{1}{2}(3-\gamma) \alpha+(\gamma-2) \beta} \neq 0$, set $\Gamma=\Gamma(\gamma, a)=\gamma\left(\frac{b^{2}}{a}\right)^{\gamma-1}, \tilde{k}=\delta^{-\frac{1}{2}(\gamma+1) \alpha+\beta \gamma} k$, and drop the tilde on $\tilde{k}$. Then, the stationary state $\left(U_{+}, V_{+}\right)$undergoes a Turing-Hopf instability at a uniquely defined critical parameter $a=a_{*}$ and a critical wavenumber $k=k_{*}$ that satisfy

$$
a_{*}^{\gamma+1} \geq g \gamma b^{2 \gamma+1} \text { and } k_{*}^{2}<b,
$$

moreover, if $c=\frac{2}{3} b \Gamma$,

$$
a_{*}^{\gamma+1}=\frac{1}{3} \gamma b^{2 \gamma+1} \text { and } k_{*}^{2}=\frac{1}{3} b
$$

and, if $c \gg 1$

$$
a_{*}^{\gamma+3}(c)=\frac{g}{\gamma} b^{2 \gamma+3} c^{2}+O(c) \text { and } k_{*}^{2}(c)=\frac{1}{2}(1-g) b+O\left(\frac{1}{c}\right) .
$$

to leading order in $c$ and $\delta$ ([59] Proposition 2).

\subsubsection{Ginzburg-Landau equation}

If

$$
\left|a-a_{*}\right|=r \epsilon^{2}
$$

and $\epsilon$ is small enough, the Ginzburg-Landau equation 16 associated to 36 has the form

$$
\mathcal{A}_{\tau}=\left(a_{1}+i a_{2}\right) \mathcal{A}_{\xi, \xi}+\left(b_{1}+b_{2}\right) \mathcal{A}+\left(L_{1}+i L_{2}\right)|\mathcal{A}|^{2} \mathcal{A}
$$

whose coefficients are functions of $b, c$, and $\gamma\left([59]\right.$, Proposition 3). The Turing-Hopf instability of $\left(U_{+}, V_{+}\right)$is supercritical if $L_{1}<0$ and subcritical if $L_{1}>0$.

The Benjamin-Feir-Newell criterion. If the Turing-Hopf bifurcation is supercritical, there exists a band of stable spatially periodic patterns if and only if

$$
1+\frac{a_{2} L_{2}}{a_{1} l_{1}}>0
$$

By means of the computing system Matematica, the authors evaluated the coefficients of $(52)$, for $\gamma=(1,2)$. These evaluations lead them to acute results:

1)For $\gamma=\{1,2\}$, the coefficients $L_{1}$ in $(52)$, is negative for all values of $b$ and $c$ up to $c \sim 10^{6}$ and $b \sim 10^{2}$, and condition (53) is satisfied. Therefore, the Turing Hopf bifurcation at $a=a_{*}$ of the stationary state $\left(U_{+}, V_{+}\right)$of $(36)$, with $C>0$ 
and $\gamma=\{1,2\}$ is supercritical ([59] Claim 1, p. 59).

2) For 36 with $C>0$ and $\gamma=\{1,2\}$, there exists a stable band of periodic patterns that appears at the Turing-Hopf instability ([59] Claim 2 p. 60).

3) For $c=0$, all coefficients of $(52)$ are real and $L_{1}=L_{1}(\gamma)$ becomes positive for large $\gamma$ and equals 0 for $\gamma \approx 13.0446$. Therefore the Turing bifurcation for 35 with $c=0$ is supercritical for $\gamma<\gamma_{s s}$, and subcritical for $\gamma>\gamma_{s s}$ ([59] proposition 4).

Remark 6. In Section 3 of [59], existence of stable patterns if $a$ is not closed to $a_{*}$ is studied. Busse Ballons (cf. Definition 5) are numerically constructed for different values of $a$ and fixed values of $b, c, \gamma$

\section{Gierer-Meinhardt system and biology}

The Gierer-Meinhardt system (cf.[27]) is one of the most popular models in biological pattern formation. Let $(S, g)$ be a compact two-dimensional Riemannian manifold without boundary.(For definitions related to Riemannian manifolds, see W. Kühnel [39]). In [57], W. Tse et al. consider the system

$$
\left\{\begin{array}{l}
A_{t}=\epsilon^{2} \Delta_{g} A-A+\frac{A^{2}}{H} \\
\tau H_{t}=\frac{1}{\beta^{2}} \Delta_{g} H-H+A^{2}
\end{array}\right.
$$

in $S$. Here, $A(p, t), H(p, t)>0$ represent the activator and the inhibitor concentrations, repectively, at a point $p \in S$ and at time $t$; their corresponding diffusivities are denoted by $\epsilon^{2}$ and $\frac{1}{\beta^{2}} ; \tau$ is the time-relaxation constant of the inhibitor and $\Delta_{g}$ is the Laplace-Beltrami operator with respect to the metric $g$. Their assumptions on the parameters $\epsilon$ and $\beta$ are: $\epsilon$ is small enough and $\lim \frac{\beta^{2}}{\epsilon^{2}}=\kappa>0$. Let $G_{0}$ be the Green function defined by

$$
\Delta_{g} G_{0}(p, q)-\frac{1}{|S|}+\delta_{p}(q)=\text { in } S, \quad \text { and } \int_{S} G_{0}(p, q) d v_{g}(q)=0
$$

$K(p)$ denote the Gauss curvature on $S$, and $w$ be the solution of the problem

$$
\Delta w-w+w^{2}=0, w>0 \text { in } \mathbb{R}^{2}, w(0)=\max _{y \in \mathbb{R}^{2}} w(y), \quad w(y) \rightarrow 0 \text { as }|y| \rightarrow \infty
$$

They introduce the function $F(p)=c_{1} K(p)+c_{2} R(p)$ where $R(p)$ denotes the diagonal of the regular part of the Green function, and

$$
c_{1}=\frac{\pi}{4} \int_{0}^{\infty}\left(\frac{\partial w}{\partial r}\right)^{2} r^{3} d r \quad c_{2}=\frac{|S| \pi}{2} \frac{\beta^{2}}{\epsilon^{2}} \int_{0}^{\infty} w^{2} r d r
$$

\subsection{Existence and stability of a single spike solution}

\subsubsection{Existence}

If $p_{0} \in S$ is a non-degenerate critical point of $F(p)$, i.e.

$$
\nabla F\left(p_{o}\right)=0, \quad \operatorname{det}\left(\nabla^{2} F\left(p_{o}\right)\right) \neq 0
$$

it is proved that problem (54) has a positive spiky steady state $\left(A_{\epsilon}, H_{\epsilon}\right)$ such that

$$
A_{\epsilon}(x)=\xi_{\epsilon}\left(w\left(\frac{x-p_{\epsilon}}{\epsilon}\right)+O\left(\epsilon^{2}\right)\right),
$$


uniformly for $x \in S$, where

$$
\xi_{\epsilon}=\frac{|S|}{\epsilon^{2} \int_{\mathbb{R}^{2}} w^{2}(y) d y}+O\left(\log \frac{1}{\epsilon}\right), \text { with } p_{\epsilon} \rightarrow p_{0} \text { as } \epsilon \rightarrow 0
$$

and

$$
H_{\epsilon}(x)=\xi_{\epsilon}\left(1+O\left(\epsilon^{2}\right)\right) \text { uniformly in } \mathrm{S}
$$

([57, Theorem 1.1).

Remark 7. It is assumed that $\xi_{\epsilon, p}$ is the height of the peak and that $p \in \overline{\Lambda_{\delta}}$ is the location of the peak, where $\Lambda_{\delta}=S \cap B_{g}\left(p_{o}, \delta\right)$, with $\delta=\epsilon^{\alpha}$, for some $0<\alpha<1 .\left(B_{g}\left(p_{o}, \delta\right)\right.$ is the ball with center $p_{o}$ and radius $\delta$, with respect to the metric $g$ ).

\subsubsection{Linear stability}

Linearizing (54) around the equilibrium states $\left(A_{\epsilon}+\phi_{\epsilon} e^{\lambda_{\epsilon} t}, H_{\epsilon}+\psi_{\epsilon} e^{\lambda_{\epsilon}} t\right.$, ) the following eigenvalue problem $L_{\epsilon}$ is obtained

$$
\left\{\begin{array}{l}
\epsilon^{2} \Delta_{g} \phi_{\epsilon}-\phi_{\epsilon}+2 \frac{A_{\epsilon}}{H_{\epsilon}} \phi_{\epsilon}-\frac{A_{\epsilon}^{2}}{H_{\epsilon}^{2}} \psi_{\epsilon}=\lambda_{\epsilon} \phi_{\epsilon} \\
\frac{1}{\tau}\left(\frac{1}{\beta^{2}} \Delta_{g} \psi_{\epsilon}-\psi_{\epsilon}+2 A_{\epsilon} \phi_{\epsilon}\right)=\lambda_{\epsilon} \psi_{\epsilon}
\end{array}\right.
$$

where $\left(A_{\epsilon}, H_{\epsilon}\right)$ is the above solution of system (54).

Definitions The solution $\left(A_{\epsilon}, H_{\epsilon}\right)$ is called linearly stable if the spectrum $\sigma\left(L_{\epsilon}\right)$ of $L_{\epsilon}$ lies in the left half plane $\{\lambda \in \mathbb{C}: \operatorname{Re}(\lambda)<0\}$. It is called linearly unstable if there exists an eigenvalue $\lambda_{\epsilon}$ of $L_{\epsilon}, \operatorname{with} \operatorname{Re}\left(\lambda_{\epsilon}\right)>0$.

Main result (Cf. [57, Theorem 1.2). Let $p_{o}$ be a non-degenerate local maximum point of $F(p)$ i.e.

$$
\nabla F\left(p_{o}\right)=0 \quad \nabla^{2} F\left(p_{o}\right) \text { is negative definite, }
$$

let $\left(A_{\epsilon}, H_{\epsilon}\right)$ be the above single peaked solution, whose peak approches $p_{o}$, then, there exists a unique $\tau_{1}>0$ such that $\left(A_{\epsilon}, H_{\epsilon}\right)$ is linearly stable [resp. unstable], if $\tau<\tau_{1}$ [resp. $\tau>\tau_{1}$ ].

\subsection{Stationary Gierer-Meinhardt system}

(Cf. [57, Section 3). The proof of the existence theorem, subsection [3.1], is based on the stationary system associated to (54) i.e.

$$
\left\{\begin{array}{c}
\epsilon^{2} \Delta_{g} A-A+\frac{A^{2}}{H}=0, \text { in } S \\
\frac{1}{\beta^{2}} \Delta_{g} H-H+A^{2}=0, \text { in } S
\end{array}\right.
$$

With the rescaled amplitudes

$$
a(p)=\frac{1}{\xi_{\epsilon, p}} A(p), \quad h(p)=\frac{1}{\xi_{\epsilon, p}} H(p)
$$

an equilibrium solution (a,h) solves the rescaled Gierer-Meinhardt system

$$
\left\{\begin{array}{l}
\epsilon^{2} \Delta_{g} a-a+\frac{a^{2}}{h}=0, \quad a>0 \text { in } S \\
\Delta_{g} h-\beta^{2} h+\beta^{2} \xi_{\epsilon, p} a^{2}=0, \quad h>0 \quad \text { in } S
\end{array}\right.
$$

with $a=O(1)$ and $h=O(1)$ 


\subsection{Existence and stability of a cluster of two spikes for the stationnary Gierer-Meinhardt system}

In [3], W. Ao et al. consider the stationnary Gierer-Meinhardt system

$$
\left\{\begin{array}{l}
\epsilon^{2} \Delta_{g} A-A+\frac{A^{2}}{H}=0, \\
D \Delta_{g} H-H+A^{2}=0
\end{array}\right.
$$

on a compact two-dimensional Riemannian manifold $(\mathcal{M}, g)$ without boundary. They assume

$$
0<\epsilon<<1, \quad 0<D<<1
$$

It is assumed that there is a non-degenerate local maximum point of the Gaussian curvature function $K(p)$ of $\mathcal{M}$ at $p_{o}=0$, i.e. $\nabla K(0)=0$ and

$$
\nabla^{2} K(0)=\left(\begin{array}{cc}
K_{11} & 0 \\
0 & K_{22}
\end{array}\right)
$$

Let $p_{o}$ be a non-degenerate local maximum point of the gaussian curvature $K(p)$ of $\mathcal{M}$. They show that, under stronger conditions on $\epsilon$ and $D$, namely

$$
0<\epsilon<<\sqrt{D}<<1, \quad 0<\sqrt{D} \log \frac{1}{\epsilon^{2} D \log \frac{\sqrt{D}}{\epsilon}}<<1,
$$

the system (67) has, at least, two different 2-spike cluster solutions $\left(A_{i}, H_{i}\right)$ for $\mathrm{i}=1,2$. Moreover, one of the solutions is stable and the other one is unstable.

\section{Harmonic Bergman spaces and reproducing kernels}

\subsection{Introduction}

This section is related to Steklov eigenproblems. Cf. F. Gazzola, H-C. Grunau, G. Sweers [26]. Let $\Omega$ be a bounded domain in $\mathbb{R}^{n}, n \geq 2$ with Lipschitz boundary $\partial \Omega$, and $a \in \mathbb{R}$. The classical Dirichlet biharmonic Steklov eigenproblem is the boundary value eigenvalue problem

$$
\Delta^{2} u=0 \text { in } \Omega, \quad u=\Delta u-a u_{\nu}=0 \text { on } \partial \Omega
$$

Here, a solution to $(71)$ is a function $u \in H^{2}(\Omega) \cap H_{0}^{1}(\Omega)$ such that

$$
\int_{\Omega} \Delta u \Delta v d x=a \int_{\partial \Omega} u_{\nu} v_{\nu} d \omega \text { for all } v \in \in H^{2}(\Omega) \cap H_{0}^{1}(\Omega)
$$

where $\nu(x)$ is the unit outward normal at $x \in \partial \Omega$. (Since $\partial \Omega$ is Lipschitzian, the tangent hyperplane and the unit outward normal $\nu=\nu(x)$ are well-defined for (a.e) $x \in \partial \Omega$ ).

\subsubsection{Orthogonal decomposition of $H^{2} \cap H_{0}^{1}(\Omega)$}

The bounded domain $\Omega$ is assumed to have a $C^{2}$-boundary, and the Hilbert space $H^{2} \cap H_{0}^{1}(\Omega)$ is endowed with the scalar product

$$
(u, v) \rightarrow \int_{\Omega} \Delta u \Delta v d x
$$


Let $Z$ be the space

$$
Z=\left\{v \in C^{\infty}(\bar{\Omega}): \Delta^{2} u=0 \text { in } \Omega, u=0 \text { on } \partial \Omega\right\},
$$

and $V$ be the completion of $Z$ with respect to the scalar product $(73)$. The following orthogonal decomposition is obtained

$$
H^{2} \cap H_{0}^{1}(\Omega)=V \oplus H_{0}^{2}(\Omega)
$$

If $v=v_{1}+v_{2}$ is the corresponding orthogonal decomposition of $v \in H^{2} \cap H^{1}(\Omega), v_{1} \in V$, and $v_{2} \in H_{0}^{2}(\Omega)$ are weak solutions of

$$
\Delta^{2} v_{1}=0 \text { in } \Omega, v_{1}=0 \text { and }\left(v_{1}\right)_{\nu}=v_{\nu} \text { on } \partial \Omega
$$

and

$$
\Delta^{2} v_{2}=\Delta^{2} v \text { in } \Omega, v_{2}=0 \text { and }\left(v_{2}\right)_{\nu}=0 \text { on } \partial \Omega
$$

([26] Theorem 3.19).

\subsubsection{The least positive eigenvalue of $(71)$}

Let $\Omega$ be a bounded domain with $C^{2}$ boundary. The least positive eigenvalue of 71 is characterised by

$$
\delta_{1}(\Omega)=\min \left\{\frac{\|\Delta u\|_{L^{2}(\Omega)}^{2}}{\left\|u_{\nu}\right\|_{L^{2}(\partial \Omega)}^{2}} ; u \in\left[H^{2}(\Omega) \cap H_{0}^{1}(\Omega)\right] \backslash H_{0}^{2}(\Omega)\right\}
$$

In [26] (Theorem 3.17), it is proved that the minimum in (78) is achieved, and, up to a multiplicative constant, the minimiser $\bar{u}$ is unique and solves $(71)$ when $a=\delta_{1}$. Furthermore, $\bar{u} \in C^{\infty}(\Omega)$, and, up to the boundary, $\bar{u}$ is as smooth as the boundary permits.

Remark 8. It is pointed out that this result is valid when $\Omega$ is a bounded domain with Lipschitz boundary which satisfied a uniform outer ball condition, where

Definition 8. A bounded domain $\Omega \subset \mathbb{R}^{n}$ satisfies an outer ball condition if for each $y \in \partial \Omega$ there exists a ball $B \subset \mathbb{R}^{n} \backslash \Omega$ such that $y \in \partial B$. If the radius of the ball $B$ is independant of $y$, we say that it satisfies a uniform outer ball condition.

\section{An alternative characterisation of $\delta_{1}(\Omega)$}

Here $\Omega$ is a bounded domain with Lipschitz boundary. Let

$$
C_{H}^{2}(\bar{\Omega})=\left\{v \in C^{2}(\bar{\Omega}) ; \Delta v=0 \text { in } \Omega\right\}
$$

equipped with the norm $\|v\|_{H}=\|v\|_{L^{2}(\partial \Omega)}$, and define

$$
\mathbf{H}=\text { the completion of } C_{H}^{2}(\bar{\Omega}) \text { with respect to the norm }\|\cdot\|_{H} \text {. }
$$

Let

$$
\sigma_{1}(\Omega)=\inf \left\{\frac{\|h\|_{L^{2}(\partial \Omega)}^{2}}{\|h\|_{L^{2}(\Omega)}^{2}} ; h \in \mathbf{H} \backslash\{0\}\right\}
$$

It is proved that $\sigma_{1}(\Omega)$ admits a minimiser. If, moreover, $\Omega$ satisfies a uniform outer ball condition, then, the minimiser is positive, unique up to a constant multiplier, and $\sigma_{1}(\Omega)=\delta_{1}(\Omega)([26]$, Theorem 3.23). 


\subsection{Subspaces of $H^{1}(\Omega)$ related to the usual Laplacian $\Delta$. Real harmonic Bergman space}

(Cf. G. Auchmuty [8]). Let $\Omega$ be a sufficiently smooth open subset of $\mathbb{R}^{N}$.

Assumption $\left(B_{1}\right)$ on $\Omega$ : $\Omega$ is a bounded connected open set whose boundary is the union of a finite number of disjoint closed Lipschitz surfaces, each surface having finite surface area.

The Hausdorff (N-1)-dimensional measure and integration with respect to this measure are denoted, respectively, by $\sigma, d \sigma$, and $\tilde{\sigma}(E)=(\sigma(\partial \Omega))^{-1} \sigma(E)$. All functions, in this section, are real valued. The following equivalent inner product on $H^{1}(\Omega)$ is introduced

$$
[u, v]_{\delta}=\int_{\Omega} \nabla u \cdot \nabla v d x+\int_{\partial \Omega} u v d \tilde{\sigma}
$$

\subsubsection{The spaces $H(\Delta, \Omega)$ and $H_{0}(\Delta, \Omega)$}

Let $H(\operatorname{div}, \Omega)$ denote the class of $L^{2}$-vector fields $\mathbf{F} \in L^{2}\left(\Omega ; \mathbb{R}^{N}\right)$, whose divergence is in $L^{2}(\Omega)$ i.e. there is a function $\varphi \in L^{2}(\Omega)$ such that

$$
\int_{\Omega} u \varphi d x=\int_{\Omega} \nabla u \cdot \mathbf{F} d x, \quad \text { for all } u \in C_{c}^{\infty}(\Omega) .
$$

Remark 9. For any integer $p \in[0, \infty], C_{c}^{p}(\Omega)$ denotes the spaces of p-continuously differentiable functions with compact support in $\Omega$. For $p=\infty, C_{c}^{\infty}(\Omega)$ is the L. Schwartz space $\mathcal{D}(\Omega)$.

\section{Definition 9.}

$$
H(\Delta, \Omega)=\left\{u \in H^{1}(\Omega) \mid \nabla u \in H(\operatorname{div}, \Omega)\right\}
$$

With the inner product

$$
[u, v]_{\delta, \Delta}=[u, v]_{\delta}+\int_{\Omega} \Delta u \Delta v d x
$$

it is a real Hilbert space.

Remark 10. If $u \in H(\Delta, \Omega)$ then $\Delta u=\operatorname{div}(\nabla u) \in L^{2}(\Omega)$.

Space $\mathcal{H}(\Omega)$

Definition 10. A function $u \in H^{1}(\Omega)$ is said to be $H^{1}$-harmonic in $\Omega$ provided

$$
\int_{\Omega} \nabla u \cdot \nabla v d x=0 \text { for all } v \in H_{0}^{1}(\Omega) .
$$

The class of all $H^{1}$-harmonic functions on $\Omega$ is denoted by $\mathcal{H}(\Omega)$. The space $H_{0}(\Delta, \Omega)$ is the orthogonal complement of $\mathcal{H}(\Omega)$, with respect to the inner product $(85)$. It is equipped with the inner product

$$
[u, v]_{\Delta}=\int_{\Omega} \Delta u \Delta v d x
$$

which generates an equivalent norm to that of 85 ( $[8]$, Lemma 3.3). 


\subsubsection{Orthogonal decomposition of $H(\Delta, \Omega)$}

The following results are stated ([8, Lemma 3.1)

1-There are closed subspaces $H_{0}^{1}(\Omega), \mathcal{H}(\Omega)$ of $H^{1}(\Omega)$, and projections $P_{0}, P_{H}$ onto these spaces such that, for all $u \in H^{1}(\Omega)$

$$
u=P_{0} u+P_{H} u \text { with }\left[P_{0} u, P_{H} u\right]_{\delta}=0
$$

2-Moreover, if $v=v_{0}+v_{H}$ is the correponding decomposition of $v \in H\left(\Delta, \Omega\right.$ ) (in particular, $\Delta v \in L^{2}(\Omega)$ ), we have

$$
\begin{gathered}
v_{0} \in H_{0}^{1}(\Omega), \Delta v_{0} \in L^{2}(\Omega), v_{0}=0 \text { on } \partial \Omega, \text { and } \\
v_{H} \in \mathcal{H}(\Omega), \Delta v_{H}=0 \text { in } \Omega, v_{H}=v \text { on } \partial \Omega .
\end{gathered}
$$

Space $\mathcal{B H}(\Omega)$

A function $b \in H(\Delta, \Omega)$ is said to be weakly biharmonic provided

$$
\int_{\Omega} \Delta u \Delta v d x=0 \text { for all } v \in \mathcal{D}(\Omega)
$$

The closed subspace of all biharmonic functions, in $H_{0}(\Delta, \Omega)$, is denoted by $\mathcal{B H}(\Omega)$.

From now on, assumption $\left(B_{1}\right)$ on $\Omega$ is replaced by :

$\left(B_{2}\right): \Omega$ satisfies $\left(B_{1}\right)$ and the normal derivative $D_{\nu}$ is a compact mapping of $H_{0}(\Delta, \Omega)$ into $L^{2}(\partial \Omega, d \sigma)$.

The Dirichlet Biharmonic Steklov (DBS) eigenproblem

Here, the DBS eigenproblem is to find solutions $(q, b) \in \mathbb{R} \times H_{0}(\Delta, \Omega)$ of the system

$$
\int_{\Omega} \Delta b \Delta v d x=q \int_{\partial \Omega} D_{\nu} b D_{\nu} v d \sigma, \quad \text { for all } v \in H_{0}(\Delta, \Omega),
$$

where $\nu$ is an outward unit normal, defined at $\sigma$ a.e. point of $\partial \Omega$. Here $q$ is the DBS eigenvalue which appears only in the boundary condition.

By means of a suitable algorithm, the author shows the existence of a maximal countable sequence of $\Delta$-orthonormal (87) ) DBS eigenfunctions $\mathcal{B}=\left\{b_{k} \mid k \geq 1\right\}$ which is a basis of the subsspace $\mathcal{B H}(\Omega)$ of $H_{0}(\Delta, \Omega)([8$, Theorem 5.3). Therefore, a biharmonic function $b$ has the spectral representation

$$
b(x)=\sum_{j=1}^{\infty}\left\langle b, b_{j}\right\rangle_{\Delta} b_{j}(x) \text { on } \Omega .
$$

Moreover, he obtains the $\Delta$-orthogonal $(\sqrt[87 p)]{)}$ decomposition

$$
H_{0}(\Delta, \Omega)=H_{00}(\Delta, \Omega) \oplus \Delta \mathcal{B H}(\Omega),
$$

where

$$
H_{00}(\Omega)=\left\{u \in H_{0}(\Delta, \Omega) \mid D_{\nu} u=0 \text { on } \partial \Omega\right\} .
$$

Let $\left\{q_{k}, k \geq 1\right\}$ be the sequence of eigenvalues corresponding to $\mathcal{B}$. The following sets are introduced:

1- Set $h_{j}=\Delta b_{j}$ and $\mathcal{B}_{H}=\left\{h_{j} \mid j \geq 1\right\} \subset L^{2}(\Omega)$ (cf. Remark 10).

2- Set $w_{j}=\sqrt{q_{j}|\partial \Omega|} D_{\nu} b_{j}, j \geq 1$, and $\mathcal{W}=\left\{w_{j}, j \geq 1\right\}$.

It is proved that $\mathcal{W}$ is an orthonormal basis for $L^{2}(\partial \Omega, d \tilde{\sigma})$. 


\subsubsection{An orthonormal basis for the real harmonic Bergman space $L_{H}^{2}(\Omega)$.}

Definition 11. The real harmonic Bergman space $L_{H}^{2}(\Omega)$ is the space of functions $u \in L^{2}(\Omega)$ that satisfy

$$
\int_{\Omega} u \Delta v d x=0 \text { for all } v \in C_{c}^{2}(\Omega)
$$

The $h_{j}=\Delta b_{j}$ for $j \geq 1$ are harmonic and $L^{2}$-orthonormal. It is proved that $\mathcal{B}_{H}$ is an ortonormal basis of $L_{H}^{2}(\Omega)$, the orthogonal projection $P_{H}$ of $L^{2}(\Omega)$ onto $L_{H}^{2}(\Omega)$ has the representation

$$
P_{H} f(x)=\sum_{j=1}^{\infty}\left\langle f, h_{j}\right\rangle h_{j}(x) \text { for all } f \in L^{2}(\Omega),
$$

([8], Theorem 6.2). Moreover, $L_{H}^{2}(\Omega)$ is a Reproducing Kernel Hilbert space with reproducing kernel

$$
R_{\Omega}(x, y)=\sum_{j=1}^{\infty} \Delta b_{j}(x) \Delta b_{j}(y), \quad x, y \in \Omega \times \Omega .
$$

\subsection{Complex harmonic Bergman spaces}

(Cf. 9] p.172). Let $\Omega$ denote an open subset of $\mathbb{R}^{n}$, and $p$ a number satisfying $1 \leq p<\infty$. The harmonic Bergman space $b^{p}(\Omega)$ is the set of complex valued harmonic functions $u$, on $\Omega$, such that

$$
\|u\|_{b^{p}}=\left(\int_{\Omega}|u|^{p} d V\right)^{\frac{1}{p}}<\infty
$$

For fixed $x \in \Omega$, the map $u \in b^{p}(\Omega) \rightarrow u(x) \in \mathbb{C}$, called point evaluation at $x$, is continuous. The space $b^{p}(\Omega)$ is a closed subspace of $L^{p}(\Omega)$, and, therefore, it is a Banach space.

Remark 11. In [9], $L^{p}(\Omega)$ is denoted $L^{p}(\Omega, d V)$.

\subsubsection{Cas $\mathrm{p}=2$, reproducing kernel of $\Omega$}

For $p=2, b^{2}(\Omega)$ is a Hilbert space with the $L^{2}(\Omega)$ inner product

$$
\langle u, v\rangle=\int_{\Omega} u \bar{v} d V
$$

As a closed subspace of $L^{2}(\Omega, d V), b^{2}(\Omega)$ is separable. For fixed $x \in \Omega$, the map $u \in b^{2}(\Omega) \rightarrow u(x) \in \mathbb{C}$ is a bounded linear functional on the Hilbert space $b^{2}(\Omega)$. By the Riesz representation theorem, there exists a unique function $R_{\Omega}(x, \cdot) \in b^{2}(\Omega)$ such that

$$
u(x)=\left\langle u, R_{\Omega}\right\rangle=\int_{\Omega} u(y) \overline{R_{\Omega}(x, y)} d V(y) .
$$

Therefore, $b^{2}(\Omega)$ is a reproducing kernel Hilbert space with reproducing kernel $R_{\Omega}$ defined on $\Omega \times \Omega$.

Remark 12. For the Riez representation theorem, see, for instance, [49] Theorem II.4, p.43.

Properties of the reproducing kernel ([9], Proposition 8.4). The reproducing kernel of $\Omega$ is real valued, if $\left(u_{m}\right)$ is an orthonormal basis of $b^{2}(\Omega)$, then

$$
R_{\Omega}(x, y)=\sum_{m=1}^{\infty} \overline{u_{m}(x)} u_{m}(y)
$$


for all $x, y \in \Omega$,

$$
R_{\Omega}(x, y)=R_{\Omega}(y, x), \text { for all } x, y \in \Omega,
$$

and

$$
\left\|R_{\Omega}(x, .)\right\|_{b^{2}}=\sqrt{R_{\Omega}(x, x)} \text { for all } x \in \Omega \text {. }
$$

Complex and real Bergman spaces have the same reproducing kernel Indeed, let $L_{H}^{2}(\Omega)$ be the real harmonic Bergman space (cf. section 4.2 where $\Omega$ satisfies conditions $\left(B_{2}\right)$. Thanks to properties of harmonic functions, if $f_{1}$ and $f_{2}$ are real-valued, and if $f=f_{1}+i f_{2}$, then $f$ belongs to $b^{2}(\Omega)$ if and only if $f_{1}, f_{2} \in L_{H}^{2}(\Omega)$. Moreover, with the notations of N. Aronszajn [7], p. 343, $b^{2}(\Omega)=\left(L_{H}^{2}(\Omega)\right)_{c}=\left\{f=f_{1}+i f_{2}: f_{1}, f_{2} \in L_{H}^{2}(\Omega)\right\}$. Therefore, the two spaces $b^{2}(\Omega)$ and $L_{H}^{2}(\Omega)$ have the same reproducing kernel given by $(98)$.

\section{Homeomorphisms of the circle. Rotation number}

There are several definitions of rotation number in the literature.

\subsection{Simple examples}

Example 1. Rotation number related to the equation $x^{\prime}=f(t, x),(t, x) \in \mathbb{R} \times \mathbb{R}$

From E.A. Coddington and N. Levinson [13]. Consider the differential equation

$$
x^{\prime}=f(t, x), \quad(t, x) \in \mathbb{R} \times \mathbb{R},
$$

where $f$ is a real continuous function, $f(t+1, x)=f(t, x+1)=f(t, x)$ and through every point of the $(t, x)$ plane there passes a unique solution of 105$)$. In $\mathbb{R}^{3}$, with rectilinear coordinates $(u, v, w)$, let $\mathcal{J}$ be the torus given by

$$
\begin{aligned}
& u=(a+b \cos 2 \pi x) \cos 2 \pi t \\
& v=(a+b \cos 2 \pi x) \sin 2 \pi t \\
& w=b \sin 2 \pi x,
\end{aligned}
$$

where $a$ and $b$ are constants with $0<b<a$. The function $f$ may be considered as a function on $\mathcal{J}$, whose points can be described by Cartesian coordinates $(t, x)$, where two points $\left(t_{1}, x_{1}\right)$ and $\left(t_{2}, x_{2}\right)$ are regarded as identical if $\left(t_{1}-t_{2}\right)$ and $\left(x_{1}-x_{2}\right)$ are integers. Through every point $P$ of $\mathcal{J}$, there exists a unique solution path $(t, \varphi(t))$ of $[105)$. Let $\varphi=\varphi(t, \eta)$ be the solution of 105 such that $\varphi(0, \eta)=\eta$, and set $\psi(\eta)=\varphi(1, \eta)$. Then $\psi$ is a homeomorphism of the real line onto itself. Let $\mathcal{C}$ be the circle on $\mathcal{J}$, defined as the set of all $(t, x)$ on $\mathcal{J}$ such that $t=0$. (The equations of $\mathcal{C}$, in $\mathbb{R}^{3}$, are $\left.v=0, u-a=b \cos 2 \pi x, v=b \sin 2 \pi x\right)$. Define the homeomorphism $T: \mathcal{C} \rightarrow \mathcal{C}$ by

$$
P=(0, \eta) \rightarrow T(P)=P_{1}=(1, \varphi(1, \eta))=(0, \psi(\eta))=(1, \psi(\eta)+n)
$$

for any integer $n$. Set

$$
\psi^{0}(\eta)=\eta, \quad \eta_{n}=\psi^{n}(\eta)=\psi\left[\psi^{n-1}(\eta)\right]
$$

and

$$
T^{0} P=P, \quad P_{n}=T^{n} P=T\left(T^{n-1} P\right),
$$

for $n=0, \pm 1, \pm 2, \ldots$ (Since $T$ is a homeomorphism, the inverse $T^{-1}$ exists). 
Definition 12. The rotation number $\rho$ of $T$ for the equation 105 is defined by

$$
\rho=\lim _{|n| \rightarrow \infty} \frac{\eta_{n}}{n}
$$

It measures the average rotation of points under $T$. The number $\rho$ is rational if and only if some power of $\mathrm{T}$ has a fixed point (cf.Theorem 2.1 de $[13]$ ).

Example 2. Rotation number for the system $\frac{d \varphi}{d t}=\Phi(\varphi, \delta), \quad \frac{d \delta}{d t}=\Theta(\varphi, \delta)$, on the torus From V.V. Nemyyskii and V.V. Stepanov [43]. The authors consider dynamical systems defined by a system of differential equations

$$
\frac{d \varphi}{d t}=\Phi(\varphi, \delta), \quad \frac{d \delta}{d t}=\Theta(\varphi, \delta)
$$

on the torus:

$$
\begin{aligned}
& u=(R+r \cos 2 \pi \delta) \cos 2 \pi \varphi \\
& v=(R+r \cos 2 \pi \delta) \sin 2 \pi \varphi \\
& w=r \sin 2 \pi \delta
\end{aligned}
$$

where $0 \leq \varphi<1, \quad 0 \leq \delta<1, \quad 0<r<R$. The functions $\Theta$ and $\Phi$ are assumed to be sufficiently smooth (for instance Lipschitz functions). Moreover, $\Phi$ is assumed to be different from 0 everywhere. By setting

$$
A(\varphi, \delta)=\frac{\Theta(\varphi, \delta)}{\Phi(\varphi, \delta)},
$$

the system 111 becomes

$$
\frac{d \delta}{d \varphi}=A(\varphi, \delta) .
$$

The trajectories, in the $(\varphi, \delta)$ plane, of equation $(114)$, yield the trajectories on the torus, if points $\left(\varphi_{1}, \delta_{1}\right)$ and $\left(\varphi_{2}, \delta_{2}\right)$ are identified when the differences $\varphi_{1}-\varphi_{2}$ and $\delta_{1}-\delta_{2}$ are integers. Let $\delta=u\left(\varphi, \delta_{0}\right)$ be the solution of 114 such that $u(0, \delta)=\delta_{0}$. If

$$
A(\varphi, \delta)=\mu
$$

where $\mu$ is a constant, the integral curves are straight lines $u\left(\varphi, \delta_{0}\right)=\delta_{0}+\mu \varphi$, whose slope is $\mu=\lim _{\varphi \rightarrow \infty} \frac{u\left(\varphi, \delta_{0}\right)}{\varphi}$.

In the general case, the rotation number $\mu$ of system 111 (or 114) is defined by

$$
\mu=\lim _{\varphi \rightarrow \infty} \frac{u\left(\varphi, \delta_{0}\right)}{\varphi} .
$$

\subsection{Rotation number of an orientation-preserving homeomorphism $f: S^{1} \rightarrow S^{1}$}

(cf. L. Wen 62 p.24). Here $S^{1}$ denotes the unit circle. Let $f: S^{1} \rightarrow S^{1}$ be an orientation-preserving homeomorphism, and $F: \mathbb{R} \rightarrow \mathbb{R}$ be a lifting of $f$. For any $t \in \mathbb{R}$, the limit

$$
\lim _{n \rightarrow \infty} \frac{F^{n}(t)-t}{n}
$$

exists, is independent of $\mathrm{t}$, and is denoted by $\rho(F)$. If $F_{1}$ and $F_{2}$ are two liftings of $f, \rho\left(F_{1}\right)-\rho\left(F_{2}\right)$ is an integer. The rotation number of $f$ is defined by $\rho(f)=\rho(F) \bmod 1$. In some sens, the rotation number of $f$ measures the average rotation of points under $\mathrm{f}$.

Remark 13. A continuous map $F: \mathbb{R} \rightarrow \mathbb{R}$ is a lifting of $f$ if $\pi \circ F=f \circ \pi$, where $\pi: t \in \mathbb{R} \rightarrow e^{2 \pi i t} \in S^{1}$ is the projection of modulo integer parts.

The homeomorphism $f$ is orientation preserving [resp. reversing] if any lifting of $f$ to $\mathbb{R}$ is strictly increasing [resp. decreasing]. 
Example 3. If $f_{\alpha}$ is a rotation by the angle $\alpha$, then $F_{\alpha}(x)=x+\alpha$ and $F_{\alpha}^{n}=x+n \alpha$, which is periodic if $\alpha$ is rational.

\subsection{Analytic reduction of analytic diffeomorphism of the circle to a rotation}

\subsubsection{Arnold's Theorem}

(V.I. Arnold [5], pp.112-115). Set

$$
\Pi_{\rho}=\{z \in \mathbb{C} ; \operatorname{Im} z \mid \leq \rho\}
$$

and, for an analytic and bounded function $a$ in this strip,

$$
\|a\|_{\rho}=\sup |a(y)|, \quad y \in \Pi_{\rho}
$$

An irrational number $\mu$ is of type $(K, \sigma), K>0, \sigma>0$, if

$$
\left|\mu-\frac{p}{q}\right| \geq \frac{K}{|q|^{2+\sigma}},
$$

for any integers $p$ and $q \neq 0$.

For a number $\mu$ of type $(K, \sigma)$, we have the "small denominator estimate" (cf [4] )

$$
\left|e^{2 \pi i k \mu}-1\right| \geq \frac{K}{2|k|^{1+\sigma}} .
$$

Then the author states the following theorem:

Theorem. There exists $\epsilon(K, \mu, \sigma)>0$, such that, if $a$ is a $2 \pi$-periodic analytic function, real on the real axis, with $\|a\|_{\rho}<\epsilon$ and such that the transformation

$$
y \rightarrow \mathbb{A}(y)=y+2 \pi \mu+a(y)
$$

is the lifting to $\mathbb{R}$ of a diffeomorphism of the circle $A$, with rotation number $\mu$ of type $(K, \sigma)$, then $\mathbb{A}$ is analytically equivalent to the rotation $R_{2 \pi \mu}$ by the angle $2 \pi \mu$ (cf. Example 3 ) i.e. there exists an analytic diffeomorphism $H: \mathbb{R} \rightarrow \mathbb{R}$ such that

$$
\mathbb{A} \circ H=H \circ R_{2 \pi \mu} .
$$

In this case, $A$ is said to be analytically conjugate to the rotation of angle $2 \pi \mu$, or analytically linearized, and $H$ is called the conjugacy or the linearization.

Remark 14. Thanks to 60, Remark 2.8, stated for fonctions of period 1 , if $\mathbb{A}$ has the more general form

$$
\mathbb{A}(x)=x+2 \pi \alpha+a(x)
$$

with the rotation number $\rho$ of type $(K, \nu)$, (where $\alpha \neq \rho)$, then if $\|\eta\|_{\sigma}=\|2 \pi(\alpha-\rho)+a\|_{\sigma} \leq \epsilon(K, \nu, \sigma)$, $\mathbb{A}$ is analytically conjugate to $R_{2 \pi \rho}$.

The following is taken from J-C. Yoccoz [53] and 63]. A number $\mu$, which satisfies condition (120), is also called Diophantine. In [53] p.57, the set of such $\mu$ is denoted by $\mathcal{C D}(K, \sigma)$. The following notations are introduced

$$
\mathcal{C D}(\sigma)=\bigcup_{K>0} \mathcal{C D}(K, \sigma) \text { and } \mathcal{C D}=\bigcup_{\sigma>0} \mathcal{C D}(\sigma)
$$




\subsubsection{The Brjuno condition and the set $\mathcal{B}$}

An irrational number $\alpha \in(0,1)$ always admits an infinite unique continuous fraction expansion (cf. H.H. Hardy and E.M. Wright [29], Theorem 170):

$$
\frac{1}{a_{1}+\frac{1}{a_{2}+\frac{1}{a_{3}+\cdots}}},
$$

also denoted

$$
\alpha=\left[a_{1}, a_{2}, \ldots\right]
$$

Then $\frac{p_{n}}{q_{n}}=\left[a_{1}, \ldots, a_{n}\right]$ is a rationnal number.

Definition 13. $\alpha$ is said to verify the Brjuno condition if and only if

$$
\sum_{n=1}^{\infty} \frac{\log q_{n+1}}{q_{n}}<\infty
$$

This is also a condition on the $\alpha \in \mathbb{R} / \mathbb{Z}$. The set of such $\alpha$ is denoted by $\mathcal{B}$.

\subsubsection{The condition and the set $\mathcal{H}$}

Let $A:(0,1) \rightarrow(0,1)$ be the map defined by

$$
A(x)=\frac{1}{x}-\left\lfloor\frac{1}{x}\right\rfloor
$$

where $\left\lfloor\frac{1}{x}\right\rfloor$ denotes the integer part of $\frac{1}{x}$, and, for $\alpha \in \mathbb{R} / \mathbb{Q}$, let $\left(\alpha_{n}\right)_{n \geq 0}$ be defined by

$$
\alpha_{0}=\alpha-\lfloor\alpha\rfloor, \quad \alpha_{n}=A^{n}\left(\alpha_{0}\right), \text { for } n>0,
$$

and $\left(a_{n}\right)_{n \geq 0}$ defined by

$$
a_{0}=\lfloor\alpha\rfloor \quad \alpha_{n-1}^{-1}=a_{n}+\alpha_{n}, \text { for all } n \geq 1
$$

Then

$$
\alpha=a_{0}+\frac{1}{a_{1}+\frac{1}{\ddots+\frac{1}{a_{n}+\alpha_{n}}}},
$$

and

$$
\frac{p_{n}}{q_{n}}=a_{0}+\frac{1}{a_{1}+\frac{1}{\ddots \cdot+\frac{1}{a_{n}}}}=\left[a_{o}, a_{1}, \ldots a_{n}\right]
$$

Let

$$
\beta_{-1}=1, \quad \beta_{n}=\prod_{j=0}^{n} \alpha_{j} \equiv(-1)^{n}\left(q_{n} \alpha-p_{n}\right) \quad \forall n \geq 0 .
$$

For $\alpha \in \mathbb{R} / \mathbb{Q}$, the Brjuno function $B: \mathbb{R} / \mathbb{Q} \rightarrow \mathbb{R}^{+} \cup\{\infty\}$ is defined by

$$
B(\alpha)=\sum_{n \geq 0} \beta_{n-1} \log \alpha_{n}^{-1}
$$




\section{Condition $\mathcal{H}$}

For $\alpha \in(0,1), x \in \mathbb{R}$, let $r_{\alpha}$ the function defined by

$$
r_{\alpha}(x)= \begin{cases}\alpha^{-1}\left(x-\log \alpha^{-1}+1\right) & \text { if } x \geq \log \alpha^{-1} \\ e^{x} & \text { if } x \leq \log \alpha^{-1}\end{cases}
$$

and set, for $\alpha \in \mathbb{R} / \mathbb{Q}$, and $k>0$, (cf.1130)

$$
\Delta_{k}(\alpha)=r_{\alpha_{k-1}} \circ \ldots \circ r_{\alpha_{0}}(0) .
$$

Let $\alpha \in \mathbb{B}$ and define, for $n \geq k \geq 0$,

$$
\mathcal{H}_{k, n}=\left\{\alpha \in \mathcal{B}, B\left(\alpha_{n}\right) \leq \Delta_{k}\left(\alpha_{n-k}\right)\right\}
$$

Definition of the set $\mathcal{H}$

$$
\mathcal{H}=\left\{\cap_{m \geq 0} \cup_{k \geq 0} \mathcal{H}_{k, k+m}\right\}=\left\{\alpha \in \mathcal{B}, \forall m \geq 0, \exists k \geq 0, B\left(\alpha_{m+k}\right) \leq \Delta_{k}\left(\alpha_{m}\right)\right\}
$$

It is proved that

$$
\mathcal{C} \subset \mathcal{B} \subset \mathcal{H}
$$

where the inclusions are proper. In [63], J-C Yoccoz proves the following result (Theorem 1.4, p.127): Let $f$ be an analytic diffeomorphism of the circle with rotation number $\alpha$. If $\alpha \in \mathcal{H}$, then $f$ is analytically conjugate to the rotation $R_{\alpha}$. Moreover, if $f \notin \mathcal{H}$, there exists an analytic diffeomorphism of the circle, $f$, with rotation number $\alpha$, which is not analytically linearizable.

Remark 15. For historical comments and the contributions of H. Poincaré (1881/6) [45], A. Denjoy (1932) [18] and M.R. Hermann (1979) 30, and (1985) 31, to the theory, see the above references and W. de Melo and S. van Strien [40].

\section{Homogenization and linear elasticity}

\subsection{Introduction}

This subsection is an introduction to the theory of homogenization. Complements will be found in the references.

\subsection{1 $\mathrm{H}$ and G-convergences}

(Cf. G. Allaire [2] ). Let $\Omega$ be a bounded open set in $\mathbb{R}^{N}$

Definition 14. Let $\mathcal{M}_{N}$ be the space of square real matrices of order $\mathrm{N}$ and define

$$
\mathcal{M}_{\alpha, \beta}=\left\{\left.M \in \mathcal{M}_{N}|M \xi \cdot \xi \geq \alpha| \xi\right|^{2}, M^{-1} \xi \cdot \xi \geq \beta|\xi|^{2}, \forall \xi \in \mathbb{R}^{N}\right\}
$$

or

$$
\mathcal{M}_{\alpha, \beta}=\left\{\left.M \in \mathcal{M}_{N}|\alpha| \xi\right|^{2} \leq M \xi \cdot \xi \leq \beta^{-1}|\xi|^{2}, \forall \xi \in \mathbb{R}^{N}\right\}
$$

and also

$$
\mathcal{M}_{\alpha, \beta}^{s}=\left\{\left.M \in \mathcal{M}_{N}^{s}|M \xi \cdot \xi \geq \alpha| \xi\right|^{2}, M^{-1} \xi \cdot \xi \geq \beta|\xi|^{2}, \forall \xi \in \mathbb{R}^{N}\right\}
$$

where $\alpha, \beta>0$ and $\alpha \beta \leq 1$, and $\mathcal{M}_{N}^{s}$ is the space of real symmetric matrices of order $\mathrm{N}$. 
Definition 15. A sequence of matrices $A^{\epsilon}(x) \in L^{\infty}\left(\Omega ; \mathcal{M}_{\alpha, \beta}\right)$ is said to converge in the sense of homogenisation, or $H$-converge, to an homogenized limit or $H$-limit matrix $A^{*} \in L^{\infty}\left(\Omega ; \mathcal{M}_{\alpha, \beta}\right)$ if, for any $f \in H^{-1}(\Omega)$, the sequence of solutions $u_{\epsilon}$ of

$$
-\operatorname{div} A^{\epsilon}(x) \nabla u_{\epsilon}(x)=f(x) \text { in } \Omega, \quad \text { and } u_{\epsilon}=0 \text { on } \partial \Omega,
$$

satisfies

$$
u_{\epsilon} \rightarrow u \text { weakly in } H_{0}^{1}(\Omega) \text { and } A^{\epsilon} \nabla u_{\epsilon} \rightarrow \text { weakly in } L^{2}(\Omega)^{N},
$$

where $u$ is the solution of the homogenized equation

$$
-\operatorname{div} A^{*}(x) \nabla u(x)=f(x) \text { in } \Omega, \quad \text { and } u=0 \text { on } \partial \Omega,
$$

(cf.[2], Definition 1.2.15 and Remark 1.2.17). This definition is justified by the following result: for any sequence $A^{\epsilon}(x)$ of matrices in $L^{\infty}\left(\Omega ; \mathcal{M}_{\alpha, \beta}\right)$ there exist a subsequence, still denoted by $A^{\epsilon}$, and an homogenized matrix $A^{*}(x) \in L^{\infty}\left(\Omega ; \mathcal{M}_{\alpha, \beta}\right)$ such that $A^{\epsilon} H$-converges to $A^{*},([2]$,Theorem 1. 2. 16)

Definition 16. A sequence of matrices $A^{\epsilon}(x) \in L^{\infty}\left(\Omega ; \mathcal{M}_{\alpha, \beta}^{s}\right)$ is said to $G$-converge, to an homogenized limit or $G$-limit matrix $A^{*} \in L^{\infty}\left(\Omega ; \mathbb{M}_{\alpha, \beta}^{s}\right)$ if, for any $f \in H^{-1}(\Omega)$, the sequence of solutions $u_{\epsilon}$ of

$$
-\operatorname{div} A^{\epsilon}(x) \nabla u_{\epsilon}(x)=f(x) \text { in } \Omega, \quad \text { and } u_{\epsilon}=0 \text { on } \partial \Omega,
$$

satisfies

$$
u_{\epsilon} \rightarrow u \text { weakly in } H_{0}^{1}(\Omega)
$$

where $u$ is the solution of the homogenized equation

$$
-\operatorname{div} A^{*}(x) \nabla u(x)=f(x) \text { in } \Omega, \quad \text { and } u_{\epsilon}=0 \text { on } \partial \Omega,
$$

(cf.[2], Definition 1.3.8). This definition is justified by the following result: for any sequence $A^{\epsilon}(x)$ of matrices in $L^{\infty}\left(\Omega ; \mathcal{M}_{\alpha, \beta}^{s}\right)$ there exist a subsequence, still denoted by $A^{\epsilon}$, and an homogenized matrix $A^{*}(x) \in L^{\infty}\left(\Omega ; \mathcal{M}_{\alpha, \beta}^{s}\right)$ such that $A^{\epsilon} H$-converges to $A^{*}$, ([2], Theorem 1.3.9)

Remark 16. For symmetric matrices, $H$ and $G$ convergences are equivalent ([2], Proposition 1.3.11).

\subsubsection{Spectral problems}

The case of abstract operators defined in different Hilbert spaces (Cf. [44, Section 1.2, p.266). Let $H_{\epsilon}, H_{0}$ be separable Hilbert spaces, $A_{\epsilon}: H_{\epsilon} \rightarrow H_{\epsilon}, 0 \leq \epsilon \leq 1, \quad A_{o}: H_{o} \rightarrow H_{o}$, be continuous linear operators, and $V$ be a subspace of $H_{o}$ with $\operatorname{Im} A_{o} \subset V \subset H_{o}$ satisfying the following conditions:

1-There exist linear continuous operators $R_{\epsilon}: H_{o} \rightarrow H_{\epsilon}$ and a constant $\gamma$ such that

$$
\left(R_{\epsilon} f, R_{\epsilon} f\right)_{H_{\epsilon}} \rightarrow \gamma(f, f)_{H_{o}}, \text { as } \epsilon \rightarrow 0, \text { for any } f \in V \text {. }
$$

2-The operators $A_{\epsilon}, A_{o}$ are positive, compact, self-adjoint and the norms $\left\|A_{\epsilon}\right\|_{B\left(H_{\epsilon}\right)}$ are bounded by constant independent of $\epsilon$.

3-For any $f \in V$,

$$
\left\|A_{\epsilon} R_{\epsilon} f-R_{\epsilon} A_{o} f\right\|_{H_{\epsilon}} \rightarrow 0 \text { as } \epsilon \rightarrow 0 .
$$


4-For each sequence $f^{\epsilon} \in H_{\epsilon}$ such that $\sup \left\|f^{\epsilon}\right\|_{H_{\epsilon}}<\infty$, one can extract a subsequence, still denoted $f^{\epsilon}$, such that, for some $w \in V$,

$$
\left\|A_{\epsilon} f^{\epsilon}-R_{\epsilon} w\right\|_{H_{\epsilon}} \rightarrow 0 \text { as } \epsilon \rightarrow 0 .
$$

The following spectral problem, for the operators $A_{\epsilon}$ and $A_{o}$, is considered

$$
\begin{aligned}
& A_{\epsilon} u_{\epsilon}^{k}=\mu_{\epsilon}^{k} u_{\epsilon}^{k}, \quad k=1,2, \ldots, \quad u_{\epsilon}^{k} \in H_{\epsilon} \\
& A_{o} u_{o}^{k}=\mu_{o}^{k} u_{o}^{k}, \quad k=1,2, \ldots, \quad u_{o}^{k} \in H_{o}
\end{aligned}
$$

with, for $\epsilon>0$ or $\epsilon=o$,

$$
\mu_{\epsilon}^{1} \geq \mu_{\epsilon}^{2} \geq \ldots \geq \mu_{\epsilon}^{k} \ldots, \quad \mu_{\epsilon}^{k}>0, \quad\left(u_{\epsilon}^{l}, u_{\epsilon}^{m}\right)_{H_{\epsilon}}=\delta_{l m}
$$

It is proved that there is a sequence $\left\{\beta_{\epsilon}^{k}\right\}$ such that $\beta_{\epsilon}^{k} \rightarrow 0$ as $\epsilon \rightarrow 0,0<\beta_{\epsilon}^{k}<\mu_{o}^{k}$, and

$$
\left|\mu_{\epsilon}^{k}-\mu_{o}^{k}\right| \leq \frac{\mu_{o}^{k} \gamma^{-1 / 2}}{\mu_{o}^{k}-\beta_{\epsilon}^{k}} \sup _{u \in N_{o}^{k},\|u\|_{o}=1}\left\|A_{\epsilon} R_{\epsilon} u-R_{\epsilon} A_{o} u\right\|_{\mathcal{H}_{\epsilon}}, \quad k=1,2, \ldots
$$

where $N_{o}^{k}$ is the eigenspace of operator $\mathcal{A}_{o}$ corresponding to the eigenvalue $\mu_{o}^{k}([44$, Theorem 1.4, p.268)

\section{Weak * convergence}

Definition 17. A sequence $\left(u_{\epsilon}\right)_{\epsilon>0}$ is said to converge weakly ${ }^{*}$ in $L^{\infty}(\Omega)$ to a limit $u$ if, for any $\phi \in L^{1}(\Omega)$, it satisfies

$$
\left.\lim _{\epsilon \rightarrow 0} \int_{\Omega} u_{\epsilon}(x) \phi(x)\right) d x=\int_{\Omega} u(x) \phi(x) d x .
$$

This definition is valid for vector valued functions.

Main result Let $A^{\epsilon} \in L^{\infty}\left(\Omega ; \mathcal{M}_{\alpha, \beta}^{s}\right)$ be a sequence of symmetric matrices which $H$-converges to an homogenized matrix $A^{*}$. Let $\rho_{\epsilon}$ be a sequence of positive functions, such that

$$
0<\rho_{-} \leq \rho_{\epsilon}(x) \leq \rho_{+}<\infty
$$

which converges weakly $*$ in $L^{\infty}(\Omega)$ to a limit $\rho(x)$. Let $\left(\lambda_{\epsilon}^{m}\right) m \geq 1$ be the eigenvalues, labeled by increasing order, and $\left(u_{\epsilon}^{m}\right)_{m \geq 1}$ be associated normalized, in $L^{2}(\Omega)$, eigenvectors of the spectral problem

$$
-\operatorname{div} A^{\epsilon}(x) \nabla u_{\epsilon}^{m}(x)=\lambda_{\epsilon}^{m} \rho_{\epsilon}(x) u_{\epsilon}^{m}(x) \text { in } \Omega \text { and } u_{\epsilon}^{m}=0 \text { on } \partial \Omega .
$$

Then, for any fixed $m \geq 1$,

$$
\lim _{\epsilon \rightarrow 0} \lambda_{\epsilon}^{m}=\lambda^{m}
$$

and, up to a subsequence, $u_{\epsilon}^{m}$ converges weakly in $H_{0}^{1}(\Omega)$, to a normalized eigenvector associated to $\lambda^{m}$, which are solutions of the homogenized eigenvalue problem

$$
-\operatorname{div} A^{*}(x) \nabla u^{m}(x)=\lambda^{m} \rho(x) u^{m}(x) \text { in } \Omega, \text { and } u^{m}=0 \text { on } \partial \Omega,
$$

and $\left(\lambda^{m}\right)_{m \geq 1}$ is the complete family of eigenvalues of $[160$, labeled in increasing order., ([2], Theorem 1.3.16). A similar result is obtained in O. A. Oleinik et al. [44, Chapter III Theorem 2.1.

\subsubsection{Periodic homogenization}

Cf. 2], Section 1.3.4. Let $Y=(0,1)^{N}$ be the unit periodic cell which is identified with the unit $N$-dimensional torus. 
Spaces $L_{\natural}^{p}(Y), 1 \leq p \leq \infty$

\section{Definition 18.}

$$
L_{\natural}^{p}(Y)=\left\{f \in L_{l o c}^{p}\left(\mathbb{R}^{N}\right) \text { such that } \mathrm{f} \text { is Y-periodic }\right\}
$$

equipped with the norm $\|f\|_{L^{p}(Y)}$.

Example Let $A(Y)$ be a matrix which belongs to $L_{\natural}^{\infty}\left(Y ; \mathcal{M}_{\alpha, \beta}\right)$. Let $\Omega$ be a bounded open set in $\mathbb{R}^{N}$. For $f \in H^{-1}(\Omega)$, let $u_{\epsilon} \in H_{o}^{1}(\Omega)$ be the unique solution of

$$
-\operatorname{div}\left(A\left(\frac{x}{\epsilon}\right) \nabla u_{\epsilon}\right)=f \text { in } \Omega, \quad u=0 \text { on } \partial \Omega
$$

Remark 17. Here the conductivity $A\left(\frac{x}{\epsilon}\right)$ of $\Omega$ shows that the domain $\Omega$ is highly heterogeneous with periodic heterogeneities of lengthscale $\epsilon$.

For each unit vector $e_{i}$, in the canonical basis of $\mathbb{R}^{N}$, let $w_{i}$ be a solution of the following problem in $Y$ :

$$
-\operatorname{div} A(y)\left(e_{i}+\nabla w_{i}(y)\right)=0 \text { in } Y, \quad y \rightarrow w_{i}(y) \text { is } Y \text {-periodic }
$$

Then, the sequence $A_{\epsilon}(x)=A\left(\frac{x}{\epsilon}\right), H$-converges to $A^{*} \in \mathcal{M}_{\alpha, \beta}$ defined by its entries

$$
A_{i j}^{*}=\int_{Y}\left[\left(A(y) \nabla w_{j}(y)\right) \cdot e_{i}+A_{i j}(y)\right] d y
$$

The starting point of the proof of this result is to write the solution $u_{\epsilon}$ of equation 162 on the form of a two-scale asymptotic expansion :

$$
u_{\epsilon}(x)=\sum_{i=0}^{+\infty} \epsilon^{i} u_{i}\left(x, \frac{x}{\epsilon}\right)
$$

where $u_{i}(x, y)$ is a function of $x$ and $y$, periodic in $y$ with period $Y$.

\subsection{Homogenization and linear elasticity}

\subsubsection{The linear elasticity operator}

In [44, the usual linear elasticity operator, in a domain $\Omega \subset \mathbb{R}^{n}$, is defined, with the usual convention of repeated indices, as

$$
L(u) \equiv \frac{\partial}{\partial x_{h}}\left(A^{h k}(x) \frac{\partial u}{\partial x_{k}}\right)
$$

where $u=\left(u_{1}, \ldots, u_{n}\right)$ is the displacement vector, and $A^{k h}(x)$ are $(n \times n)$-matrices whose elements $a_{i j}^{h k}(x)$ are bounded measure functions such that

$$
a_{i j}^{h k}(x)=a_{j i}^{k h}(x)=a_{h j}^{i k}(x), \quad x \in \Omega,
$$

and

$$
\kappa_{1} \eta_{i h} \eta_{i h} \leq a_{i j}^{h k}(x) \eta_{i h} \eta_{j k} \leq \kappa_{2} \eta_{i h} \eta_{i h}, \quad x \in \Omega
$$

for any symmetric matrix with real elements $\left\{\eta_{i h}\right\}$, with positive constants $\kappa_{1}, \kappa_{2}$.

Remark 18. Are also associated to the above operator 166 the matrix $e(u)$ whose elements are $e_{i j}(u)=\frac{1}{2}\left(\frac{\partial u_{i}}{\partial x_{j}}+\frac{\partial u_{j}}{\partial x_{i}}\right)$ which is called the strain tensor, and the Neumann boundary operator

$$
\sigma(u)=\nu_{h} A^{h k}(x) \frac{\partial u}{\partial x_{k}}(x), \quad x \in \partial \Omega
$$

where $\nu=\left(\nu_{1}, \ldots, \nu_{n}\right)$ is the unit outward normal to $\partial \Omega$. 


\subsubsection{Homogenization in perforated domains}

Notations and assumptions (Cf. 44, chapter 1). Let $\omega$ be a smooth unbounded domain of $\mathbb{R}^{n}$ invariant under the shifts by any $z=\left(z_{1}, z_{2}, \ldots, z_{n}\right) \in \mathbb{Z}^{n}, Q=\left\{x \mid 0<x_{j}<1, j=1,2, \ldots, n\right\}, \epsilon G=\left\{x \mid \epsilon^{-1} x \in G\right\}$ satisfying the conditions: $\omega \cap Q$ is a domain with Lipschitz boundary, the sets $Q \backslash \bar{\omega}$ and $V_{\delta} \cap \partial Q$ consist of a finite number of Lipshitz domains separated from each other and from the edges of $Q$ by a positive distance, where $V_{\delta}$ is a $\delta$ - neigborhhod $\left(\delta<\frac{1}{4}\right)$ of $\partial Q$.

Definition 19. Let $\Omega$ be a bounded smooth domain in $\mathbb{R}^{n}$. A domain $\Omega^{\epsilon}$ is of type I if

$$
\Omega \epsilon=\Omega \cap \epsilon \omega
$$

The boundary of $\Omega_{\epsilon}$ can be represented as

$$
\partial \Omega_{\epsilon}=\Gamma_{\epsilon} \cup S_{\epsilon} \text { where } \Gamma_{\epsilon}=\partial \Omega \cap \epsilon \omega, \quad S_{\epsilon}=\left(\partial \Omega_{\epsilon}\right) \cap \Omega .
$$

Example (Cf. [44, p. 119-134). Let $\Omega_{\epsilon}$ be a domain of type (170). Consider the following mixed problem:

$$
L_{\epsilon}\left(u_{\epsilon}\right)=\frac{\partial}{\partial x_{i}}\left(A^{i j}\left(\frac{x}{\epsilon}\right) \frac{\partial u_{\epsilon}}{\partial x_{j}}\right)=f_{\epsilon} \text { in } \Omega_{\epsilon}
$$

where the coefficients $a_{k l}^{i j}$ of matrices $A^{i j}$ are sufficiently smooth, satisfont 167)-168 and are 1-periodic in $\xi$, with the boundary conditions

$$
u_{\epsilon}=\Phi_{\epsilon} \text { on } \Gamma_{\epsilon}, \quad \sigma_{\epsilon}\left(u_{\epsilon}\right)=\nu_{i} A^{i j} \frac{\partial u_{\epsilon}}{\partial x_{j}}=0 \text { on } S_{\epsilon} .
$$

Here, $f_{\epsilon} \in L^{2}\left(\Omega_{\epsilon}\right)$ and $\Phi_{\epsilon} \in H^{1}\left(\Omega_{\epsilon}\right)$. The homogenized operator corresponding to $L_{\epsilon}$ has the form

$$
\mathcal{L} \equiv \frac{\partial}{\partial x_{i}}\left(\mathcal{A} i j \frac{\partial}{\partial x_{j}}\right)
$$

where the coefficients matrices $\mathcal{A}^{i j}(i, j=1, \ldots, n)$ are given by the formula

$$
\mathcal{A}^{i j}=(\operatorname{mes} Q \cap \omega)^{-1} \int_{Q \cap \omega}\left(A^{i j}(\xi)+A^{i k}(\xi) \frac{\partial N^{j}(\xi)}{\partial \xi_{k}}\right) d \xi
$$

and matrices $N^{j}(\xi)$ are 1-periodic in $\xi$ with $\int_{Q \cap \omega} N^{j}(\xi) d \xi=0$ and are solutions of the following boundary problem:

$$
\frac{\partial}{\partial \xi_{k}}\left(A^{k j}(\xi) \frac{\partial N^{q}}{\partial \xi_{j}}\right)=-\frac{\partial}{\partial \xi_{k}} A^{k q}(\xi) \text { in } \omega
$$

with

$$
\sigma\left(N^{q}\right)=-\nu_{k} A^{k q}(\xi) \text { on } \partial \omega
$$

Let $u_{o}(x)$ be a weak solution of the problem

$$
\mathcal{L}\left(u_{o}\right)=f_{o} \text { in } \Omega, \quad u_{o}=\Phi_{o} \text { on } \partial \Omega
$$

where $f_{0} \in H^{1}(\Omega)$, and $\Phi_{o} \in H^{3}(\Omega)$. If $f_{\epsilon}$ is the restriction to $\Omega_{\epsilon}$ of $f_{o}$, and $\Phi_{\epsilon}$ is the restriction to $\Gamma_{\epsilon}$ of $\Phi_{o}$, then 178 is the homogenized system corresponding to system 172 and 173 and $\left\|u_{\epsilon}-u_{o}\right\|_{L^{2}\left(\Omega_{\epsilon}\right)} \rightarrow 0$ as $\epsilon$ tends to 0 .

\subsection{Homogenization and Steklov eigenproblems}

In [12, Chechkina, D'Apice and De maio consider a smooth domain $\Omega$ in $\mathbb{R}^{d}, d \geq 2$, whose boundary is $\partial \Omega=\gamma_{1} \cup \gamma_{\epsilon} \cup \Gamma_{\epsilon}$. Here, $\epsilon$ is a small, positive, parameter, $\Gamma_{\epsilon}$ consists of $N_{\epsilon}$ sets whose diameter is less than or equal to $\epsilon$ and the distance 
between then is greater than or equal to $2 \epsilon$, and $\gamma_{\epsilon}=\partial \Omega \backslash\left(\gamma_{1} \cup \Gamma_{\epsilon}\right)$. The boundary value problem for the elasticity system (E):

$$
L_{k}\left(u_{\epsilon}\right)=\frac{\partial}{\partial x_{i}}\left(a_{k l}^{i j} \frac{\partial u_{\epsilon}^{l}}{\partial x_{j}}\right)=0 \text { in } \Omega, k=1, \ldots, d
$$

and

$$
\begin{gathered}
u_{\epsilon}=0 \text { on } \gamma_{1} \cup \gamma_{\epsilon}, \\
\sigma\left(u_{\epsilon}\right)=A^{i j}(x) \frac{\partial u_{\epsilon}}{\partial x_{j}} \nu_{i}=g(x) \text { on } \Gamma_{\epsilon}
\end{gathered}
$$

is considered. Here, the elements $a_{k l}^{i j}$ of the $d \times d$-matrices $A^{i j}$ are bounded measurable functions, with

$$
a_{k l}^{i j}(x)=a_{l k}^{j i}(x)=a_{i l}^{k j}(x), \quad \kappa_{1} \xi_{k i} \xi_{k i} \leq a_{k l}^{i j}(x) \xi_{k i} \xi_{l j} \leq \kappa_{2} \xi_{k i} \xi_{k i}, \quad x \in \Omega
$$

where $\kappa_{1}, \kappa_{2}$ are constants $>0, \nu=\left(\nu_{1}, \nu_{2}, . . ; \nu_{d}\right)$ is an outward normal vector to the boundary $\partial \Omega, g(x) \in\left(L^{2}(\partial \Omega)\right)^{d}$. Let $H^{1}\left(\Omega, \gamma_{1} \cup \gamma_{\epsilon}\right)$ be the completion of the space of functions $v \in C^{\infty}(\bar{\Omega}), v=0$ on $\gamma_{1} \cup \gamma_{\epsilon}$, with respect to the norm

$$
\|v\|_{H^{1}(\Omega)}=\left(\int_{\Omega}\left(v^{2}+|\nabla v|^{2}\right) d x\right)^{\frac{1}{2}}
$$

The solution $u_{\epsilon}$ of system (E) is in the space $\left(H^{1}\left(\Omega, \gamma_{1} \cup \gamma_{\epsilon}\right)\right)^{d}$. The following results are proved:

R1-the solutions $u_{\epsilon}$ of system (E) are uniformly bounded with respect to $\epsilon$, in $H^{1}(\Omega)$ ([12, Lemma 3) i.e. each $u_{\epsilon}^{i}$ is bounded in $H^{1}(\Omega)$

$\mathrm{R} 2-$

$$
\int_{\Omega}\left|u_{\epsilon}\right|^{2} d x \leq C|\ln \epsilon|^{-\delta}, 0<\delta<2-\frac{2}{d} .
$$

([12, Theorem 3). In particular $u_{\epsilon} \rightarrow 0$, in $L^{2}(\Omega)$, as $\epsilon \rightarrow 0$.

The Steklov-type problem

$$
L\left(u_{\epsilon}^{n}\right)=\frac{\partial}{\partial x_{i}}\left(a_{k l}^{i j} \frac{\partial u_{\epsilon}^{l}}{\partial x_{j}}\right)=0 \text { in } \Omega, k=1, \ldots, d
$$

with the boundary conditions

$$
u_{\epsilon}^{n}=0 \text { on } \gamma_{1} \cup \gamma_{\epsilon},
$$

and

$$
\sigma\left(u_{\epsilon}^{n}\right)=A^{i j}(x) \frac{\partial u_{\epsilon}}{\partial x_{j}} \nu_{i}=\lambda_{\epsilon}^{n} u_{\epsilon}^{n} \text { on } \Gamma_{\epsilon}
$$

is considered. The set $\left\{\lambda_{\epsilon}^{n}\right\}, n=1,2, \ldots$ is the set of eigenvalues such that $\lambda_{\epsilon}^{1} \leq \lambda_{\epsilon}^{2} \leq \ldots \leq \lambda_{\epsilon}^{n} \leq \ldots$, where the eigenvalues repeat according to their multiplicities.

The following estimate is established, for $\epsilon$ sufficiently small, and $N_{\epsilon}=O\left(|\ln |^{\left(1-\frac{\delta}{2) d-1}\right)}\right.$

$$
\lambda_{\epsilon}^{n} \geq C|\ln \epsilon|^{\delta},
$$

where $C$ is a constant independent of $\epsilon,([12$, Theorem 5). The proof is based on an adaptation of the general abstract result (subsection 6.1.2), thanks to the above results $\mathrm{R} 1$ and $\mathrm{R} 2$.

\section{Maxwell's equations and electromagnetisme}

\subsection{Spaces $H(\operatorname{div} ; \Omega), H(\operatorname{curl} ; \Omega)$}

Cf. H. M. Yin et al. 64] and D. R. Adhikari et al. [1]. Let $\Omega$ be a bounded Lipschitz domain in $\mathbb{R}^{3}$ and $p \geq 2$ 
Definition 20. We recall that, for a three dimensional vector distribution, $v \in\left(\mathcal{D}^{\prime}(\Omega)\right)^{3}$,

$$
\operatorname{curl}(v)=\nabla \times v, \quad \text { and } \quad \operatorname{div}(v)=\nabla \cdot v
$$

\section{Definition 21.}

$$
H_{p}(\operatorname{div} ; \Omega)=\left\{u \in\left(L^{p}(\Omega)\right)^{3} \mid \nabla \cdot u \in\left(L^{p}(\Omega)\right)^{3}\right\}
$$

equipped with the graph norm

$$
\|u\|_{H_{P}(\operatorname{div} ; \Omega)}=\left(\|u\|_{\left(L^{p}(\Omega)\right)^{3}}^{p}+\|\nabla \cdot u\|_{\left(L^{p}(\Omega)\right)^{3}}^{p}\right)^{1 / p}
$$

Definition 22 .

$$
H_{p}(\operatorname{curl} ; \Omega)=\left\{v \in\left(L^{p}(\Omega)\right)^{3} \mid \nabla \times v \in\left(L^{p}(\Omega)\right)^{3}\right\}
$$

equipped with the graph norm. This space is the space of finite energy solutions of Maxwell's equations.

Remark 19. In P. Monk [1], the above spaces are defined for $p=2$ and respectively denoted by $H($ div; $\Omega$ ) and $H(\operatorname{curl} ; \Omega)$. The space $H_{o}(\operatorname{curl} ; \Omega)$ is the closure of the L. Scwartz's space $(\mathcal{D}(\Omega))^{3}$ in $H(\operatorname{curl}(\Omega))$.

Definition 23. The space of surface tangential vector fields in $L^{2}(\partial \Omega)$ is defined by

$$
L_{t}^{2}(\partial \Omega)=\left\{u \in\left(L^{2}(\partial \Omega)\right)^{3} \mid \quad \nu . u=0 \text { a.e. on } \partial \Omega\right\}
$$

equipped with the norm of $\left(L^{2}(\partial \Omega)\right)^{3}$, where $\nu$ is the unit outward normal to $\Omega$.

\subsection{Maxwell's equations}

Cf. [41. The electric and magnetic field vectors are respectively denoted by $\mathcal{E}$ and $\mathcal{H}$. The. vector functions electric displacement and magnetic induction are denoted, respectively, by $\mathcal{D}$ and $\mathcal{B}$. They are functions of the position $x \in \mathbb{R}^{3}$ and time $t$, and are related by Maxwell's equations:

$$
\begin{gathered}
\frac{\partial \mathcal{B}}{\partial t}+\nabla \times \mathcal{E}=0 \\
\nabla \cdot \mathcal{D}=\rho \\
\frac{\partial \mathcal{D}}{\partial t}+\nabla \times \mathcal{H}=-\mathcal{J} \\
\nabla \cdot \mathcal{B}=0,
\end{gathered}
$$

where $\rho$ is a scalar charge density function and $\mathcal{J}$ is the current vector density function.

\subsubsection{Time-harmonic Maxwell's system}

If the radiation has a frequency $\omega>0$, in time, the electromagnetic field is said to be time-harmonic, provided

$$
\begin{aligned}
\mathcal{E}(x, t) & =\Re(\exp (-i \omega t) \hat{E}(x)) \\
\mathcal{D}(x, t) & =\Re(\exp (-i \omega t) \hat{D}(x)) \\
\mathcal{H}(x, t) & =\Re(\exp (-i \omega t) \hat{H}(x)) .
\end{aligned}
$$


And, also

$$
\begin{aligned}
\mathcal{B}(x, t) & =\Re(\exp (-i \omega t) \hat{B}(x)), \\
\mathcal{J}(x, t) & =\Re(\exp (-i \omega t) \hat{J}(x)), \\
\rho(x, t) & =\Re(\exp (-i \omega t) \hat{\rho}(x)) .
\end{aligned}
$$

Substituting these relations into Maxwell's equations (subsection 7.2, leads to the time harmonic Maxwell's system:

$$
\begin{gathered}
-i \omega \hat{B}+\nabla \times \hat{E}=0 \\
\nabla \cdot \hat{D}=\hat{\rho} \\
-i \omega \hat{D}+\nabla \times \hat{H}=-\hat{J} \\
\nabla \cdot \hat{B}=0,
\end{gathered}
$$

\subsection{The cavity problem.}

Cf. [41. In this section, the domain $\Omega \subset \mathbb{R}^{3}$ is bounded and simply connected. Its boundary $\partial \Omega$ consists of at most two connected components $\Sigma$ and $\Gamma$. Define the space:

$$
X=\left\{u \in H(\operatorname{curl} ; \Omega) \quad \nu \times u=0 \text { on } \Gamma \text { and } u_{T} \in\left(L^{2}(\Sigma)\right)^{3} \text { on } \Sigma\right\}
$$

where $\nu$ is the unit outward normal to $\Omega$ and $u_{T}=(\nu \times u) \times u$ on $\partial \Omega$. We also need the following definitions:

Definition 24. The electric permittivity, the magnetic permeability and the conductivity are denoted by $\epsilon_{o}, \mu_{o}$, and $\sigma$, respectively. Then the relative permittivity and relative permeability are denoted by $\epsilon_{r}$ and $\mu_{r}$.

Set

$$
E=\epsilon_{o}^{1 / 2} \hat{E}, \quad H=\mu_{o}^{1 / 2} \hat{H}
$$

and let $F$ be a given current density. The elimination of $H$ in the system (204)-(207) leads to the problem: find the time electric field E corresponding to $F$ such that ([41], Section 1.4)

$$
\nabla \times\left(u_{r}^{-1} \nabla \times E\right)-\kappa^{2} \epsilon_{r} E=F \text { in } \Omega
$$

with the boundary conditions

$$
\nu \times E=0 \text { on } \Gamma \text { and } \mu_{r}^{-1}(\nabla \times E) \times \nu-i \kappa \lambda E_{T}=g \text { on } \Sigma,
$$

where $\mathrm{g}$ is a given tangential vector field on $\Sigma, \kappa=\omega \sqrt{\epsilon_{o} \mu_{o}}$ and the impedance $\lambda$ is a positive function on the surface of the material.

\subsubsection{Variational formulation}

Let $(u, v)$ and $\langle u, v\rangle$ be the inner products in $\left(L^{2}(\Omega)\right)^{3}$ and $\left(L^{2}(\Sigma)\right)^{3}$, respectively and set

$$
a(u, v)=\left(\mu_{r}^{-1} \nabla \times u, \nabla \times v\right)-\kappa^{2}\left(\epsilon_{r} u, v\right)-i \kappa\left\langle u_{T}, v_{T}\right\rangle
$$

for all $u, v \in X$. Then the variational cavity problem is to find $E \in X$ such that

$$
a(E, \Phi)=(F, \Phi)+\left\langle g, \Phi_{T}\right\rangle
$$


for all $\Phi \in X$ (41] p. 82). Next, it is proved ([41], Theorem 4.17) that, under suitable additional conditions on the data, problem 213 has a unique solution $E \in X$ for any value of $\kappa>0$. Moreover there is a constant $C>0$, independent of $E, F$ and $g$ but depending on $\kappa$, such that

$$
\|E\|_{X} \leq C\left(\|F\|_{\left(L^{2}(\Omega)\right)^{3}}+\|g\|_{\left.\left(L^{2}(\Sigma)\right)^{3}\right)}\right.
$$

\subsubsection{Eigenvalues problem}

(Cf. 41, Section 4.7). In this subsubsection , $\Sigma=\emptyset$ (therefore $\mathrm{g}=0$ and $S=\Gamma$ ) and $\epsilon_{r}$ is real. The cavity eigenvalue problem is: find non-trivial pairs $E \in H_{o}(\operatorname{curl} ; \Omega)$ and $\kappa \in \mathbb{R}$ such that

$$
\left(\mu_{r}^{-1} \nabla \times E, \nabla \times \Phi\right)=\kappa^{2}\left(\epsilon_{r} E, \Phi\right) \quad \text { for all } \Phi \in H_{o}(\operatorname{curl} ; \Omega) .
$$

Main result: There exist an infinity of eigenfunctions $E$ for the eigenvalue $\kappa=0$. And there is an infinite discrete set of eigenvalues $\kappa_{j}>0, j=1,2, \ldots$ and corresponding eigenfunctions $E_{j}$, with $0<\kappa_{1} \leq \kappa_{2} \leq \ldots, \lim _{j \rightarrow \infty} \kappa_{j}=\infty$.

\subsection{The Bean model in superconductivity}

Cf. L. Prigozhin [46] and [47. The superconductor occupies a three dimensional domain $\Omega \subset \mathbb{R}^{3}$. Let $\omega$ denote the exterior space. In Maxwell's equations, the displacement current,very small, can be omitted.These equations read:

$$
\begin{gathered}
\frac{\partial B}{\partial t}+\operatorname{curl} E=0 \\
J=\operatorname{curl} H
\end{gathered}
$$

where $J$ is the current density and $B=\mu_{o} H$ where $\mu_{o}$ is the permeability of the vacuum. The density of the external current $J_{e}(x, t)$ which satisfies the condition $\operatorname{div} J_{e}=0$ is known:

$$
J_{e}=\operatorname{curl} H \quad \text { in } \omega .
$$

Only the case where the vectors of current density $J$ and electric field $E$ are collinear is considered. This is the case for two dimensional problems and three dimensional problems with axial symmetry. Jointly with the above Maxwell's equations, the Bean model presented in [47] consists of the following equations (Ohm's law)::

$$
E=\rho J \text { in } \Omega, \quad \rho(x, t) \geq 0
$$

where $\rho$ is an unknown function and $J$ is the effective resistivity,

$$
|\operatorname{curl} H| \leq J_{c}
$$

where the critical value $J_{c}$ is constant in the Bean model, moreover

$$
|\operatorname{curl} H|<J_{c} \Rightarrow \rho=0,
$$

with the initial condition:

$$
B(0, x)=B_{o}(x) \text { with } \operatorname{div} B_{o}=0
$$

and the boundary conditions: the tangential component $H_{\tau}$ of $H$ is assumed to be continuous i.e.

$$
\left[H_{\tau}\right]=0 \text { on } \Gamma
$$


where [.] denotes the jump across the boundary, and

$$
|H| \rightarrow 0 \text { as }|x| \rightarrow \infty
$$

Let

$$
V=\left\{\varphi(x, t) \mid \operatorname{curl} \varphi=0 \text { in } \omega,\left[\varphi_{\tau}\right]=0 \text { on } \Gamma\right\},
$$

The following variational relation is valid for all $\varphi \in V$ :

$$
\mu_{o} \int_{0}^{T} \int_{\mathbb{R}^{3}} \frac{\partial H}{\partial t} \cdot \varphi+\int_{0}^{T} \int_{\Omega} \rho \operatorname{curl} H . \operatorname{curl} \varphi=0
$$

To sum up, the above Bean model now consists of equations: 218, 219-224) and 226.

Let $H_{e}$ be the quasistationary external magnetic field induced by the external current in the absence of the superconductor, i.e. the unique solution of the problem

$$
\operatorname{curl} H_{e}=J_{e}, \quad \operatorname{div} H_{e}=0, \quad\left|H_{e}\right| \rightarrow 0 \text { as }|x| \rightarrow \infty \text {, }
$$

$h=H-H_{e}$ and $K(h)$ be the set of functions

$$
K(h):\left\{\varphi \in V|| \operatorname{curl} \varphi \mid \leq J_{c}\left(h+H_{e}\right) \text { in } \Omega\right\}
$$

The above Bean model is equivalent to the problem: find $h \in K(h)$ such that

$$
\left(\partial\left(h+H_{e}\right) / \partial t, \varphi-h\right) \geq 0, \text { for any } \varphi \in K(h) \text { and }\left.h\right|_{t=0}=h_{0}=\frac{B_{o}}{\mu_{o}}-\left.H_{e}\right|_{t=0} .
$$

Existence and uniqueness of solution to this problem is proved ([47, p.193).

The variational formulation of the Bean model [229] is used to solve the problem numerically ([47] Section 4), and examples are presented.

\subsection{Bean's critical state type II superconductors}

In this subsection, $\Omega$ is a bounded, simply connected domain in $\mathbb{R}^{3}$ with boundary $\partial \Omega \in C^{1,1}$ and $Q_{T}=\Omega \times(0, T], T>0$. The degenerate evolution system studied by H-M Yin et al. 64] is:

$$
\left.H_{t}+\nabla \times|\nabla \times H|^{p-2} \nabla \times H\right]=F(x, t), \quad \nabla \cdot H=0, \quad(x, t) \in Q_{T}
$$

with the conditions

$$
\nu \times H(x, t)=0, \quad(x, t) \in \partial \Omega \times[0, T]
$$

and

$$
H(x, 0)=H_{o}(x), \quad x \in \Omega,
$$

where $\mathrm{H}$ is the magnetic field, $p \geq 2$ is fixed and $\nu$ is the outward normal to $\partial \Omega$. In type-II superconductors, the electromagnetic field concerns an alloy-type conductive medium where the displacement current is small in comparaison with eddy currents $J=\sigma E$ (Ohm's law). Let $E$ be the electric field and $J$ be the current density. In Bean's model, there exists a critical current $J_{c}$ such that $|J| \leq J_{c}$ and

$$
|E|=0 \text { if }|J|<J_{c} \text { and }|E|=[0, \infty) \text { if }|J|=J_{c} .
$$

Under suitable conditions on the data $F$ and $H_{o}$, the authors prove the existence of a weak solution to the system $230-232$, whose limit $H^{(\infty)}$, as $p \rightarrow \infty$, satisfies

$$
H_{t}^{(\infty)}-\nabla \times\left[a(x, t) \nabla \times H^{(\infty)}\right]=F, \quad(x, t) \in Q_{t},
$$


in the sense of distributions, where $a(x, t)$ is a nonnegative, bounded and measurable function. Moreover

$$
\text { supp } a(x) \subset N_{t} \text { for a.e. every } t \in(0, T]
$$

where $N_{t}=\left\{(x, t) \in Q_{T}\right\}$. Therefore, $H^{(\infty)}$ is a solution to the Bean's model.

\section{Pseudospectra and non hermitian, one dimensional, quantum mechan- ics}

As usual, operator means, always, linear operator.

Pseudospectra are related to closed operators.

Notation In a Banach space $X$, the norm is denoted by $\|\cdot\|$, or, if a confusion is possible, by $\|\cdot\|_{X}$.

\subsection{Introduction: closed operators}

(Cf. L.N. Trefethen, M. Embree, [56]). Let $(X,\|\cdot\|)$ be a Banach space, $\mathcal{B}(X)$ be the set of bounded operators on $X$ and $\mathcal{C}(X)$ be the set of closed operators on $X$. (An operator $A$ is closed provided that, if $\left\{u_{k}\right\}$ is a sequence in the domain $D(A)$ of $A$, converging to a limit $u \in X$, and if $\left\{A u_{k}\right\}$ converges to a limit $v \in X$, then $u \in D(A)$ and $A u=v$.)

Remark 20. For a closed unbounded operator $A$ in $X$, its domain $\mathrm{D}(\mathrm{A})$ is different from $X$, but the domain $D(A)$ of a bounded operator is the whole of $X$.

\subsubsection{Invertibility and perturbation of closed operators}

If $A \in \mathcal{C}(X)$ has a bounded inverse $A^{-1}$, then, for any $E \in \mathcal{B}(X)$, with $\|E\|<1 /\left\|A^{-1}\right\|, A+E$ has a bounded inverse $(A+E)^{-1}$ satisfying

$$
\left\|(A+E)^{-1}\right\| \leq \frac{\left\|A^{-1}\right\|}{1-\|E\|\left\|A^{-1}\right\|} .
$$

Conversely, for any $\mu>1 /\left\|A^{-1}\right\|$ there exists $E \in \mathcal{B}(X),\|E\|<\mu$, such that $(A+E) u=0$ for some nonzero $u \in X$. Cf. [56], Theorem 4.1 and [36] Theorem 1.16, p. 196.

Given $A \in \mathcal{C}(X)$, the resolvent set $\rho(A)$ is the set of $z \in \mathbb{C}$ for which the inverse $(z-A)^{-1}$ exists and is in $\mathcal{B}(X)$. The spectrum $\sigma(A)$ of $A \in \mathcal{C}(X)$ is the complement of $\rho(A)$ in $\mathbb{C}$, i.e. $\sigma(A)=\mathbb{C} / \rho(A)$, with the convention that, for $z \in \sigma(A),\left\|(z-A)^{-1}\right\|=+\infty$.

\subsubsection{Metrics for closed operators in a Hibert space}

Product metric (Cf. L. Schwartz [51] Chapitre VII). Let $\left(E_{1}, d_{1}\right)$ and $\left(E_{2}, d_{2}\right)$ two metric spaces. It is possible to introduce, on the product $E_{1} \times E_{2}$, the following equivalent metrics $\delta=\operatorname{Max}\left(d_{1}, d_{2}\right)$ and $d_{1}+d_{2}$, respectively defined by

$$
\delta\left(\left(x_{1}, x_{2}\right),\left(y_{1}, y_{2}\right)\right)=\operatorname{Max}\left(d_{1}\left(x_{1}, y_{1}\right), d_{2}\left(x_{2}, y_{2}\right)\right)
$$

and

$$
\left(d_{1}+d_{2}\right)\left(\left(x_{1}, x_{2}\right),\left(y_{1}, y_{2}\right)\right)=d_{1}\left(x_{1}, y_{1}\right)+d_{2}\left(x_{2}, y_{2}\right)
$$


Remark 21. Let $H, K$ be two Hilbert spaces. The product $H \times K$ with the scalar product

$$
\left(\left(h_{1}, k_{1}\right),\left(h_{2}, k_{2}\right)\right)_{H \times K}=\left(h_{1}, h_{2}\right)_{H}+\left(k_{1}, k_{2}\right)_{K}
$$

is a Hilbert space. On the other hand, the topological direct sum $H \oplus K$ is the Hilbert space $\{h \oplus k=(h, k) \in H \times K\}$ with the scalar product

$$
\left\langle h_{1} \oplus k_{1}, h_{2} \oplus k_{2}\right\rangle=\left(h_{1}, h_{2}\right)_{H}+\left(k_{1}, k_{2}\right)_{K} .
$$

and the map $(h, k) \in H \times K \rightarrow h \oplus k \in H \oplus K$ is an isomorphism (cf. [19, p.112)

Metrics for closed linear subspaces of a Hilbert space (Cf. Gohberg-Krein [28] and Cordes-Labrousse [14]). Let $H$ be a Hilbert space and $\mathfrak{S}, \mathfrak{T}$ two closed subspaces of $H$.

$$
d(\mathfrak{T}, \mathfrak{S})=\sup _{x \in \mathfrak{S},\|x\|=1} d(x, \mathfrak{T})+\sup _{x \in \mathfrak{T},\|x\|=1} d(x, \mathfrak{S})
$$

defines a metric on the totality of closed linear subsets of $H$. Equivalent metrics are defined by

$$
g(\mathfrak{T}, \mathfrak{S})=\left\|P_{\mathfrak{T}}-P_{\mathfrak{S}}\right\|,
$$

where $P_{\mathfrak{S}}, P_{\mathfrak{T}}$ are the orthogonal projections on $\mathfrak{S}$ and $\mathfrak{T}$, respectively, and by

$$
\theta(\mathfrak{T}, \mathfrak{S})=\operatorname{Max}\left\{\sup _{x \in \mathfrak{S},\|x\|=1} d(x, \mathfrak{T}), \sup _{x \in \mathfrak{T},\|x\|=1} d(x, \mathfrak{S})\right\}
$$

Metrics for closed operators Let $A$ be a closed linear operator (or, simply, closed operator) in the Hilbert space $H$, with domain $D(A)$, and $\mathfrak{S}(A)$ be the graph of $A$ i. e. the set of pairs $(u \in D(A), A u)$ in $H \times H$. With the product metric, $H \times H$ is a Hilbert space (cf. subsection 8.1.2), and it is well known that A is closed in $H$, if and only if its graph $\mathfrak{S}(A)$ is a closed linear manifold in $H \times H$ (cf. Kato 36. p. 164) or, in terms of topological direct sum, if $\mathfrak{S}(A)$ is a closed linear subspace of the direct sum $\mathfrak{h}=H \oplus H$.

Definition 25. ([14], Definition 3.1). If $A$ and $B$ are closed operators acting in $H$, then the metrics $d(A, B)$ and $g(A, B)$ are defined by

$$
d(A, B)=d(\mathfrak{S}(A), \mathfrak{S}(B)), \quad g(A, B)=g(\mathfrak{S}(A), \mathfrak{S}(B))
$$

and

$$
\theta(A, B)=\theta(\mathfrak{S}(A), \mathfrak{S}(B))
$$

\subsection{Pseudospectra}

\subsubsection{Definition of the $\epsilon$-pseudospectrum $\sigma_{\epsilon}(A)$}

Let $A \in \mathcal{C}(X)$ and $\epsilon>0$ be arbitrary.

In [56], p. 31, the authors give the following equivalent definitions.

Definition 26. The $\epsilon$-pseudospectrum of $A$ is the set of $z \in \mathbb{C}$ satisfying any of the conditions

$$
\begin{gathered}
\left\|(z-A)^{-1}\right\|>\epsilon^{-1}, \\
z \in \sigma(A+E) \text { for some } E \in \mathcal{B}(X) \text { with }\|E\|<\epsilon,
\end{gathered}
$$




$$
z \in \sigma(A) \text {, or }\|(z-A) u\|<\epsilon \text { for some } u \in D(A) \text { with }\|u\|=1 \text {. }
$$

If $z$ and $u$ satisfy the last equation they are called $\epsilon$-pseudoeigenvalue and $\epsilon$-pseudoeigenvector, respectively, for the operator $A$. The pseudospectra of $A$ is the family

$$
\left\{\sigma_{\epsilon(A)}\right\}_{\epsilon>0}=\overline{\bigcup_{\|E\|<\epsilon}(\sigma(A+E)}
$$

Importance of the pseudospectra. In accordance with formula 249, the notion of pseudospectra provides information about the instability mentionned in remark 26. The size of the pseudospectral regions provides a clear indication of the instability of typical Hamiltonians in quantum mechanic.

\section{Trivial pseudospectra}

A closed operator $T$ is said to have a trivial pseudospectra if, for some positive constant $\kappa$,

$$
\left\|(T-z)^{-1}\right\| \leq \frac{\kappa}{\operatorname{dist}(z, \sigma(T))} \quad \text { for all } z \in \mathbb{C} / \sigma(T)
$$

(cf [32], section 2.3).

\subsubsection{Examples}

Example 4. The virtual eigenvalues of J. Arazy and L. Zelenko [6] are in the pseudospectra of $(-\Delta)^{\ell}$, in $X=L^{2}\left(\mathbb{R}^{d}\right), \ell \in \mathbb{N}, 2 \ell \geq d$. Indeed, they consider the operator (see also [33], p.132):

$$
\begin{gathered}
H_{\gamma}=(-\Delta)^{\ell}+\gamma V(x), \quad D\left(H_{\gamma}\right)=\left\{u \in L^{2}\left(\mathbb{R}^{d}\right) \mid u \in W_{2, l o c}^{2 l}\left(\mathbb{R}^{d}\right), H_{\gamma} u \in L^{2}\left(\mathbb{R}^{d}\right)\right\}, \\
\gamma \in \mathbb{R}, \quad x \in \mathbb{R}^{d} \rightarrow V(x) \in \mathbb{R},
\end{gathered}
$$

where $V(x) \geq 0$ is assumed to be continuous and to satisfy $\lim _{|x| \rightarrow \infty} V(x)=0$. Here $A=(-\Delta)^{\ell}$ is self-adjoint and, therefore, closed, the multiplication operator $V .=V(x)$ is bounded and belongs to $\mathcal{B}(X)$. Their virtual eigenvalues are the eigenvalues of $H_{\gamma}$, where

$$
\|\gamma V\|_{\mathcal{B}(X)}=|\gamma|\|V\|_{\mathcal{B}(X)} \leq \epsilon
$$

as soon as

$$
|\gamma| \leq \frac{\epsilon}{\|V\|_{\mathcal{B}(X)}}
$$

Therefore, for $\epsilon>0, \sigma\left(H_{\gamma}\right)$ is the $\epsilon$-pseudospectra of $(-\Delta)^{l}$ when the inequality 254 is satisfied.

Example 5. (Cf [56] p.35-37). Let $A$ be the operator acting in $X=L^{2}(0, d), d>0$, whose domain is

$$
D(A)=\left\{u \in L^{2}(0, d), u(d)=0, u \text { is absolutely continuous (ac) }\right\},
$$

such that

$$
A u=\frac{d u}{d x} \text { and } u(d)=0, \quad \text { for } u \in D(A) .
$$

We recall that a function $F(x)$ is absolutely continuous in an interval $(a, b)$ if it is the indefinite integral of a function $f \in L_{l o c}^{1}$ (cf. [54] Section 11.7). The spectrum $\sigma(A)$ is empty, since, for $z \in \mathbb{C}, u(d)=e^{z d} \neq 0$. Nevertheless, the pseudospectra of $A$ are "enormous". The resolvent $(z-A)^{-1}$ exists as a bounded operator, and, for any $z \in \mathbb{C}$,

$$
u(x)=(z-A)^{-1} v(x)=\int_{x}^{d} e^{z(x-s)} v(s) d s, \quad x \in(0, d) .
$$


The equation (257) means that $(z-A)^{-1} v(x)$ is the restriction to $(0, d)$ of the convolution product $v * g$ where $v$ and $g$ are regarded as functions in $L^{2}(-\infty,+\infty)$ with

$$
g(x)=e^{z x} \text { for } x \in[-d, 0], 0 \text { otherwise } .
$$

By means of the Fourier transform in $L^{2}(\mathbb{R}), 257$ leads to

$$
\|u\| \leq\|v * g\|=\|\widehat{v * g}\|=\|\hat{v}\|\|\hat{g}\| \leq\|v\| \sup _{\omega \in \mathbb{R}}|\hat{g}(\omega)|=\|v\| \frac{e^{-d R e}-1}{|\operatorname{Re} z|}
$$

where $\|\cdot\|$ denotes the norm in $L^{2}(-\infty,+\infty)$. Then

$$
\left\|(z-A)^{-1}\right\| \leq \frac{1}{\operatorname{Re} z}
$$

for $\operatorname{Rez}>0$, and

$$
\|(z-A)^{-1}||=\frac{e^{d|\operatorname{Re} z|}}{2|\operatorname{Re} z|}+\mathcal{O}\left(\frac{1}{|\operatorname{Re} z|}\right),
$$

for Rez $<0$. These results imply ([56], Theorem 5.1) that the pseudospctra of $A$ are half-planes of the form

$$
\sigma_{\epsilon}(A)=\left\{z \in \mathbb{C}: \operatorname{Re} z<c_{\epsilon}\right\}
$$

with

$$
c_{\epsilon} \sim \begin{cases}(\log \epsilon / d) & \text { if } \epsilon \rightarrow 0 \\ \epsilon & \text { if } \epsilon \rightarrow \infty\end{cases}
$$

Example 6. The ghost solution of D. Domokos and P. Holmes (Cf. [21]). In [56], p. 98-99, this ghost solution is presented in the following way. The author considers the linear differential equation

$$
A u=u^{\prime}+x u=0
$$

acting on sufficiently smooth functions in $L^{2}(-L,+L)$ and associated to the boundary conditions

$$
u(-L)=u(L)=0
$$

The function

$$
u(x)=e^{-\frac{x^{2}}{2}}-e^{-\frac{L^{2}}{2}}
$$

satisfies the boundary conditions 265 and the equation

$$
A u=u^{\prime}(x)+x u(x)=x e^{-\frac{L^{2}}{2}}
$$

i.e. 264 for all $\mathrm{x}$, up to an error no greater than $L e^{-\frac{L^{2}}{2}}$. Therefore

$$
|A u| \leq L e^{-\frac{L^{2}}{2}}
$$

and 0 belongs to the $\epsilon$-pseudospectrum of $A$ for a value of $\epsilon$ that decreases exponentially as $L \rightarrow \infty$.

\section{Example 7. The non-self-adjoint (NSA) harmonic oscillator}

\section{The harmonic oscillator}

The harmonic oscillator is the closure, in $L^{2}(\mathbb{R})$, of the operator $H_{a}$ defined by

$$
\left(H_{a} f\right)(x)=-f^{\prime \prime}(x)+a x^{2} f(x)
$$


for $f$ in the L. Schwartz's space $\mathcal{S}(\mathbb{R})$, with $a>0$. The operator $H_{a}$ is essentialy self-adjoint on $\mathcal{S}$ (i.e. its closure is self-adjoint in $L^{2}(\mathbb{R})$ ), and the resolvent operators are compact. Moreover, the spectrum of $H_{a}$ is

$$
\left\{(2 n+1) a^{1 / 2}: n=0,1, \ldots\right\}
$$

each eigenvalue $\lambda$ is of multiplicity 1 , and the corresponding eigenfunctions are

$$
\phi_{n}(x)=H_{n}\left(a^{1 / 4} x\right) e^{-a^{1 / 2} x^{2} / 2}
$$

where $H_{n}$ is the hermite polynomial of degree $n$. After normalization, the eigenfunctions provide a complete orthonormal set in $L^{2}(\mathbb{R})$.

\section{The non-self-adjoint (NSA) harmonic oscillator}

\section{Reminder: Definitions}

Cf. [17]. Let $X$ be a Hilbert space with inner product $(f, g) \rightarrow\langle f, g\rangle$. A sequence $\left\{x_{j}\right\}$, in $X$, is a normalized basis if it is a basis with $\left\|x_{j}\right\|=1$ for each $j$.

An unconditional basis is a basis with the property that every permutation of the sequence is also a basis.

A sequence $\left\{f_{n}\right\}_{n=1}^{\infty}$, in $X$, is said to be an Abel-Lidskii basis in $X$, if it is a part of a biorthogonal pair $\left\{f_{n}\right\},\left\{\phi_{n}\right\}$ such that, for all $f \in X$, one has

$$
f=\lim _{\epsilon \rightarrow 0} \sum_{n=1}^{\infty} e^{-\epsilon n}\left\langle f, \phi_{n}\right\rangle f_{n}
$$

Remark 22. If $X$ is a Hilbert space, a sequence $\left\{x_{j}\right\}$ is an unconditional basis if and only if there exists a bounded invertible operator $S$ on $X$ such that $\left\{e_{j}=S^{-1} x_{j}\right\}$ is a complete orthonormal set in $X$. It is also called Riesz basis.

The NSA is the operator $H_{a}$ defined by 269 where $a$ is allowed to be complex. In this case, the eigenvalues are complexe, but they are given by the same formula (270). It is proved, in E.B. Davies [17, Corollary 14.5.2, that the sequence of eigenfunctions $\phi_{n}$ cannot be an Abel-Lidskii basis and Theorem 14.5.4, that, if $0<\theta<\arg (a)$, the resolvent $R_{r, \theta}=\left(r e^{i \theta} I-H_{a}\right)^{-1}$ is such that $\left\|R_{r, \theta}\right\|$ diverges at a super-polynomial rate, as $r \rightarrow \infty$ and if $\arg (a)<\theta<2 \pi$, then $\left\|R_{r, \theta}\right\| \rightarrow 0$ as $r \rightarrow \infty$. Therefore, given $\epsilon>0$, there exists $r_{o}(\epsilon)$ such that, for $r>r_{o},\left\|R_{r, \theta}\right\|>\epsilon^{-1}$ i.e. the $\epsilon$-pseudospectra of $H_{a}$ is $\left\{z=r e^{i \theta}, 0<\theta<\arg (a), r>r_{o}\right\}$.

Remark 23. In [15], E.B. Davies considers the harmonic oscillator (269), when $a$ is complexe, with $R e a>0$ and Im $a>0$. He introduces two real positive parameters $\eta$ and $\alpha$ and constructs a family of pseudoeigenfunctions $f_{\eta}$ concentrated around the point $x_{0}=\alpha \eta$, associated to the pseudoeigenvalues $z_{\eta}=\eta^{2}+a \alpha^{2} \eta^{2}-i a \alpha$ such that

$$
\lim _{\eta \rightarrow+\infty}\left\|\left(H_{a}-z_{\eta}\right)^{-1}\right\|=+\infty
$$

He also proves that the eigenfunctions of $H_{a}$ do not form an unconditional basis in $L^{2}(\mathbb{R})$.

Example 8. In [32, R. Henry and D.Krejčiřík consider, in $L^{2}(\mathbb{R})$, the operator

$$
H=-\frac{d^{2}}{d x^{2}}+i \operatorname{sign}(x),
$$

with domain

$$
D(H)=W^{2,2}(\mathbb{R}) .
$$

It is closed and densely defined, but, neither self-adjoint nor normal. However it satisfies

$$
H^{*}=\mathcal{T} H \mathcal{T}, \quad H^{*}=\mathcal{P} H \mathcal{P}, \quad \text { and }[H, \mathcal{P} \mathcal{T}]=0(\mathcal{P} \mathcal{T} \text {-symmetry })
$$


where $H^{*}$ denotes the adjoint of $H$ and $\mathcal{T}$ and $\mathcal{P}$ are, respectively, defined by $\mathcal{T} \psi=\bar{\psi},(\mathcal{P} \psi)(x)=\psi(-x)$. Its numerical range $\operatorname{Num}(H)$ (i.e. the set of all complex numbers $(\psi, H \psi)$ with $\psi \in D(H)$ and $\|\psi\|=1$ ), is

$$
\operatorname{Num}(H)=\overline{\mathcal{S}}, \quad \text { where } \mathcal{S}=[0,+\infty)+i(-1,1)
$$

Moreover,

$$
\sigma(H)=\sigma_{e s s}(H)=[0,+\infty)+i\{-1,+1\} .
$$

They show that $H$ cannot have trivial pseudospectra. For that, they set $z=\tau+i \delta$ and they construct a function $f_{0}$ such that

$$
\left\|(H-z)^{-1}\right\| \geq \frac{\left\|(H-z)^{-1} f_{0}\right\|}{\left\|f_{0}\right\|} \geq \phi(\tau, \delta) \sim \frac{\tau}{\sqrt{1-\delta^{2}}},
$$

as $\tau \rightarrow \infty$, where $\phi(\tau, \delta)$ is a suitable function. For $z$ real, positive $(\delta=0)$

$$
\left\|(H-z)^{-1}\right\| \geq \frac{\left\|(H-z)^{-1} f_{0}\right\|}{\left\|f_{0}\right\|} \geq \phi(\tau, 0) \sim \tau,
$$

and $\operatorname{dist}(z, \sigma(H))=1$. The equation 280 shows that, for any positive constant $C$, there exists a $z_{0} \in \mathbb{C} /(\sigma(H)$, real, positive, such that

$$
\left\|\left(H-z_{0}\right)^{-1}\right\|>\frac{C}{\operatorname{dist}\left(z_{0}, \sigma(H)\right)}
$$

ever, $H$ has a non-trivial pseudospectra. Indeed, thanks to 280 , given $\epsilon>0$, there exist $z \in \mathbb{C} / \sigma(H)$ such that $\left\|(H-z)^{-1}\right\|>\epsilon^{-1}$.

Remark 24. In the references of this section, the authors present nice figures of the pseudospectra.

\subsection{Non-hermitian one dimensional quantum mechanics}

\subsubsection{Introduction to quantum mechanics}

In one dimensional motion of a single particule restricted to motion along a line, M. Schechter [50] postulates: there is a function $\psi(x, t)$ of position $x \in \mathbb{R}$ at the time $t$ such that the probability that the particule is in an interval $I$, at the time $t$, is given by

$$
\int_{I}|\psi(x, t)|^{2} d x
$$

$\psi(x, t)$ is called the state function, and satisfies $\int_{\infty}^{\infty}|\psi(x, t)|^{2} d x=1$, forall t. Set (with the notations of [50]) $p=m \frac{d x}{d t}$ where $\mathrm{m}$ is the mass of the particule, and define the operator $L$ by

$$
L \psi=-i \hbar \frac{\partial \psi}{\partial x}
$$

where $\hbar$ is the Planck's constant. (No confusion is possible between the square $L^{2}$ of the operator $L$ ans the space $\left.L^{2}(\mathbb{R})\right)$. The total energy of the particule is the sum of the kinetic energy $T=\frac{1}{2} m v^{2}=\frac{p^{2}}{2 m}$ and the potential energy $V: x \in \mathbb{R} \rightarrow \mathbb{R}$. The corresponding energy-operator or hamiltonian is the operator

$$
H=\frac{1}{2 m} L^{2}+V
$$

Remark 25. If $H$ is the generator of a $C_{o}$ semi-group $e^{-t H}$ on $L^{2}(\mathbb{R}), \psi(x, t)=e^{-t H} \psi(x, 0)$. Therefore, it will be convenient to estimate $\left\|e^{-t H}\right\|$. 
Definition 27. Let $w$ be a measurable quantity which can take on the values $w_{1}, \ldots, w_{N}$ and suppose the probability that $w$ takes the value $w_{k}$ is $P_{k}, k=1, \ldots, N$. The quantity

$$
\bar{w}=w_{1} P_{1}+\ldots+w_{N} P_{N}
$$

is called the average value or he mathematical expectation of $w$.

In [50], this definition is justified by a Feller's theorem namely: If a sequence of identical experiments is performed and the values $S_{1}, \ldots, S_{n}, \ldots$ are observed (The numbers $S_{n}$ are among the values $w_{1}, \ldots, w_{N}$ ), then the average value

$$
\frac{S_{1}+\ldots+S_{n}}{n}
$$

converges to $\bar{w}$, in the sense of probability, as $n \rightarrow \infty([22])$.

The kinetic energy of the particule is $E=\frac{p^{2}}{2 m}$. The average value of $E$ is

$$
\bar{E}=(H(\psi), \psi)
$$

Any quantity, that can be measured, is called an observable. In formula (287), $\bar{E}$ is an observable.

Remark 26. Importance of the spectrum :An observable can assume values only in the spectrum of its corresponding operator. Moreover, even in the cases in which the eigenfunctions can be determined explicitely, they often do not form a basis. This is closely related to a high degree of instability of the eigenfunctions under small perturbations of the operator (cf [16], Abstract).

\subsubsection{Examples}

This paragraph is devoted to examples of pseudospectra related to models of non-hermitian quantum mechanics (cf. Krejčiřík et al.) 38

Example 9. The rotated harmonic oscillator that is the quantum hamiltonian of the harmonic oscillator

$$
H f=-f^{\prime \prime}+x^{2}
$$

See operator 269 , with $a=1$.

Remark 27. It is proved, in [38], that the shifted harmonic oscillator

$$
-\frac{d^{2}}{d x^{2}}+(x+i)^{2}, \text { on } L^{2}(\mathbb{R})
$$

has the same spectrum as the above $H$, but large pseudospectra in parabolic regions of the complex plane ([38, Theorem 7).

Example 10. The imaginary Airy operator. It is the non-self-adjoint operator

$$
H=-\frac{d^{2}}{d x^{2}}+i x, \quad \text { on } L^{2}(\mathbb{R}),
$$

with domain $D(H)=\left\{\psi \in W^{2,2}(\mathbb{R}) ; x \psi \in L^{2}(\mathbb{R})\right\}$. The spectrum of $H$ is empty. But, its pseudospectra $\sigma_{\epsilon}(H)$ is not trivial. There exist constants $C_{1}, C_{2}$ such that, for all $\epsilon>0$,

$$
\sigma_{\epsilon}(H) \supseteq\left\{z \mid \Re z \geq C_{1} \text { and } \Re z \geq C_{2}\left(\log \frac{1}{\epsilon}\right)^{2 / 3}\right.
$$


Example 11. The imaginary cubic oscillator:

$$
H=-\frac{d^{2}}{d x^{2}}+i x^{3}, \quad \text { on } L^{2}(\mathbb{R}),
$$

with domain $D(H)=\left\{\psi \in W^{2,2}(\mathbb{R}) ; x^{3} \psi \in L^{2}(\mathbb{R})\right\}$. Its spectum is composed of an infinite sequence of discrete real eigenvalues and the eigenfunctions form a complete set in $L^{2}(\mathbb{R})$ (cf. [52]). Its pseudospectra $\sigma_{\epsilon}(H) 1$ is described as follows: for any $\delta>0$, there exist constants $C_{1}, C_{2}>0$ such that, for all $\epsilon>0$,

$$
\sigma_{\epsilon}(H) \supseteq\left\{z \in \mathbb{C}|| z \mid \geq C_{1} \text { and }|\arg z|<\left(\frac{\pi}{2}-\delta\right) \text { and }|z| \geq C_{2}\left(\log \frac{1}{\epsilon}\right)^{6 / 5}\right\}
$$

Example 12. An advection-diffusion operator:

$$
H=-\frac{d^{2}}{d x^{2}}+\frac{d}{d x} \text { on } L^{2}(\mathbb{R})
$$

with domain $D(H)=W^{2,2}(\mathbb{R})$. The diffusion term $-d^{2} / d x^{2}$ is the free Hamiltonian in quantum mechanics, self-adjoint on $W^{2,2}(\mathbb{R})$. The spectrum $\sigma(H)$ of $H$ is the parabola $\partial \Sigma$ :

$$
\sigma(H)=\left\{z \in \mathbb{C} \mid \Re z \geq 0 \text { and }|\Im z|^{2}=\Re z\right\} .
$$

Since $H$ is a normal operator, its pseudo-spectra are trivial. Neverthless, the spectrum of the self-adjoint free Hamiltonian $H_{s a}=-\frac{d^{2}}{d x^{2}}+\frac{1}{4}=\Omega H \Omega^{-1}$ with (cf. (312) $\Omega=e^{-x / 2}$, with domain $W^{2,2}(\mathbb{R}), \sigma\left(H_{s a}\right)=(1 / 4, \infty)$, differs from the complex parabolic spectrum of $H$.

Remark 28. In [38, the authors point out the differences between the above operator 294 and the convectiondiffusion operator:

$$
H^{(L)}=-\frac{d^{2}}{d x^{2}}+\frac{d}{d x} \text { on } L^{2}[0, L]
$$

with the Dirichlet boundary conditions $u(0)=u(L)=0$. In this case,

$$
\sigma\left(H^{(L)}\right)=\sigma\left(H_{s a}^{(L)}\right)=\left\{\left(\frac{\pi k}{L}\right)^{2}+\frac{1}{4} \mid k=1,2, \ldots\right\}
$$

Example 13. (Cf. [48]). Consider the operator

$$
H=: H^{([0, d])}=\frac{d^{2}}{d x^{2}}+\frac{d}{d x} \text { on } L^{2}[0, d],
$$

with the Dirichlet boundary conditions $u(0)=u(d)=0$. The following related operators will be considered: $H^{([0, \infty))}$, acting in $L^{2}[0, \infty)$ with the boundary condition $u(0)=0$, and $H^{(-\infty,+\infty))}$ acting in $L^{2}(-\infty,+\infty)$ with no boundary condition.

\section{Notations}

$$
S=\{\alpha \in \mathbb{C}:-1 \leq \Re \alpha<0\} ; \quad \Delta_{\epsilon}=\{\lambda \in \mathbb{C}:|\lambda| \leq \epsilon\}
$$

and

$$
P=\left\{\lambda \in \mathbb{C}: \lambda=\alpha^{2}+\alpha, \Re \alpha=0\right\}, \quad \Pi=\left\{\lambda \in \mathbb{C}: \lambda=\alpha^{2}+\alpha,-1 \leq \Re \alpha<0\right\}
$$

\section{Spectra of the above operators}

$$
\sigma(H)=-\frac{1}{4}-\frac{\pi^{2} n^{2}}{d^{2}}, n=1,2, \ldots \quad \sigma\left(H^{-\infty,+\infty}\right)=P \quad \sigma\left(H^{([0, \infty))}\right)=\Pi
$$

Pseudospectra For each $\epsilon>0$, the $\epsilon$-pseudospectra of $H, H^{(-\infty,+\infty)}$, and $H^{[0, \infty)}$ satisfy

$$
\sigma_{\epsilon}(H) \subseteq \Pi+\Delta_{\epsilon}
$$

and

$$
\sigma_{\epsilon}\left(H^{(-\infty,+\infty)}\right)=P+\Delta_{\epsilon}, \quad \sigma_{\epsilon}\left(H^{([0,+\infty))}\right)=\Pi+\Delta_{\epsilon}
$$




\subsubsection{Time evolution}

(cf. Remark 25. In this subsection, $H$ is a closed linear operator in a Hilbert space, which generates a $C_{O}$ semi-group (cf. [55]).

\section{Definitions}

Definition 28. The spectral abcissa of $H, \alpha_{H}$, is the $\underset{\lambda \in \sigma(H)}{\sup }$ of the real part or of the absolute value of the spectrum of $H$.

Definition 29. The $\epsilon$ pseudospectral abcissa of $H, \alpha_{\epsilon}(H)$ is the $\underset{\lambda \in \sigma_{\epsilon}(H)}{\sup }$ of the real part or of the absolute value of the $\epsilon$-pseudo spectrum of $H$.

Definition 30. The numerical abcissa of $H, w(H)$, is

$$
w(H)=\sup _{z \in W(H)} \Re z
$$

where $W(H)=\{(H u, u) ; u \in D(H),\|u\|=1\}$, is the numerical range of $H$.

Definition 31. The the growth abcissa of $H$ is

$$
\gamma(H)=\lim _{t \rightarrow \infty} t^{-1} \log \left\|e^{t H}\right\|
$$

Main results 1) For any $H$ satisfying the above assumption, we have:

$$
\gamma(H)=\lim _{\epsilon \rightarrow 0} \alpha_{\epsilon}(H)
$$

2) If $H$ is normal we have

$$
\left.\|\exp (t H)\|=e^{t \alpha(H}\right), \forall t>0
$$

If $H$ is not normal

$$
e^{t \alpha(H)} \leq\|\exp (t H)\| \leq+\infty
$$

But, when $H$ has a basis of eigenvectors, the last inequality, in (308), may be affined in terms of the condition number of this basis.

\subsubsection{Symmetrizability}

Definition 32. An operator $\mathcal{L}$ is symmetrizable if it is similar, by a diagonal similarity transformation, to a self-adjoint operator with the same real eigenvalues.

For example, in (cf [48]), the authors consider the convection-diffusion operator

$$
\mathcal{L} u=u^{\prime \prime}+u^{\prime}, \quad u(0)=u(d)=0
$$

acting in $L^{2}[0, d]$, with domain

$$
D(\mathcal{L})=\left\{u \in C[0, d] \mid u\left(0=u(d)=0, \mathrm{u} \text { has a second derivative } u " \in L^{2}(0, d)\right\} .\right.
$$


The spectrum $\sigma(\mathcal{L})$ and the spectral abcissa $\alpha(\mathcal{L})$ are,

$$
\lambda_{n}=-\frac{1}{4}-\frac{\pi^{2} n^{2}}{d^{2}}, \quad n=1,2,3, \ldots, \quad \alpha(\mathcal{L})=-\frac{1}{4}-\frac{-\pi^{2}}{d^{2}}
$$

and the corresponding eigenfunctions

$$
u_{n}(x)=e^{-x / 2} \sin (\pi n x / d)
$$

form a basis. Set $u(x)=e^{-\frac{x}{2}} v(x)$.Then $\mathcal{L} u=u^{\prime \prime}+u^{\prime}=e^{-\frac{x}{2}}\left(v^{\prime \prime}-\frac{1}{4} v\right)$. Finally,

$$
\mathcal{L} u=\mathcal{M K} v=\mathcal{M K} \mathcal{M}^{-1} u
$$

where $\mathcal{K}: v \rightarrow v^{\prime \prime}-\frac{1}{4 v}$ is self adjoint, $\mathcal{M}: v \rightarrow \mathcal{M} v=e^{-\frac{x}{2}} v$, is a diagonal operator with $\|\mathcal{M}\|=1$ and $\left\|\mathcal{M}^{-1}\right\|=e^{\frac{d}{2}}$, and therefore $\kappa(\mathcal{M})=\|\mathcal{M}\|\left\|\mathcal{M}^{-1}\right\|=e^{d / 2}$.

Remark 29. The number $\kappa(\mathcal{M})$ is the condition number of the symmetrizing transformation, but, it is also equal to the condition number of the basis $(312)$ of eigenfunctions of $\mathcal{L}$. In this case, the inequality $(308)$ can be written

$$
e^{t \alpha(\mathcal{L})} \leq\left\|e^{t \mathcal{L}}\right\| \leq \kappa(\mathcal{M}) e^{t \alpha(\mathcal{L})}
$$

i.e.

$$
e^{-t / 4-t \pi^{2} / d^{2}} \leq\left\|e^{t \mathcal{L}}\right\| \leq e^{d / 2-t / 4-t \pi^{2} / d^{2}}
$$

(48], p.1646).

\section{Spectral flow}

There are several definitions of spectral flow in the literature.

\subsection{A roughly speaking description}

In [23], P.M. Fitzpatrick et al. present the following description of the spectral flow. Let $H$ be a real separable Hilbert space, $\Phi_{0}(H)$ be the space of all (linear) Fredholm operators of index 0 acting on $H$, (i.e. its kernel $N(T)$ has finite dimension $n u l(T)$, its range $R(T)$ is closed and has a finite codimension $\operatorname{def}(T)$; the index of $\mathrm{T}$ is $i(T)=n u l(T)$-def $(T)$ ) and $\Phi_{S}(H)$ the subset of $\Phi_{0}(H)$ of self-adjoint and, therefore closed, operators. Thanks to subsection 8.1 .2 , $\Phi_{S}(H)$ is a topological space. Let $I=[a, b]$ be a real interval. For a path i.e. a continuous map $L: \lambda \in I \rightarrow L_{\lambda} \in \Phi_{S}(H)$, the spectral flow $\operatorname{sf}(L, I)$ is the number of negative eigenvalues of $L_{a}$ that become positive as the parameter $\lambda$ travels from $a$ to $b$ minus the number of positive eigenvalues of $L_{a}$ that become negative.

A similar description was presented and justified by K. Furutani and N. Otsuki in [25] , when $H$ is a separable complex Hilbert space and $\Phi_{S}(H)$ is the space of bounded Fredholm, self-adjoint operators with index 0 .

\subsection{Spectral flow via the Cayley transform}

(Cf. B. Booss-Bavnbek et al. [10]). Let $H$ be a separable complex Hilbert space with the scalar product ( , ). The authors denote by $\mathcal{C}^{s a}$ the space of closed, densely defined operators $T$ acting in $H$, that are self-adjoint, and by $\mathcal{C F}^{s a}$ the subspace of $\mathcal{C}^{s a}$ that are Fredholm .

\section{Several topologies have been introduced on $\mathcal{C}^{s a}$.}




\subsubsection{The gap topology}

Let $T \in \mathcal{C}^{s a}$, and $\mathrm{P}$ denotes the orthogonal projection onto the graph of $T$ in $H \times H$. The gap metric is $\delta\left(T_{1}, T_{2}\right)=$ $\left\|P_{1}-P_{2}\right\|$ (where $\|$.$\| denotes the norm in the space \mathcal{B}$ of bounded operators acting in $H$ )

\subsubsection{The "Cayley transform" metric}

For a densely defined operator $T$ in $H$, the Cayley transform $\kappa$ is defined by

$$
T \rightarrow \kappa(T)=(T-i)(T+i)^{-1}
$$

Let $\mathcal{U}$ be the subspace of $\mathcal{B}$ of unitary operators $H \rightarrow H$. It is proved that

$$
\kappa\left(\mathcal{C F}^{s a}\right)=\{u \in \mathcal{U} \mid(U+I) \text { is Fredholm and }(U-I) \text { is injective }\}=:_{\mathcal{F}} \mathcal{U}_{i n j}
$$

moreover the metric $\tilde{\delta}$, on $\mathcal{C F}^{s a}$, defined by

$$
\tilde{\delta}\left(T_{1}, T_{2}\right)=\left\|\kappa\left(T_{1}\right)-\kappa\left(T_{2}\right)\right\|
$$

is equivalent to the metric $\delta$.

\subsubsection{The metric $\gamma$}

On $\mathcal{C}^{s a}$, the metric $\gamma$, defined by

$$
\gamma\left(T_{1}-T_{2}\right)=\left\|\left(T_{1}+i\right)^{-1}-\left(T_{2}+i\right)^{-1}\right\|
$$

is uniformly equivalent to the gap metric, and $\tilde{\delta}\left(T_{1}, T_{2}\right)=\frac{1}{2} \gamma\left(T_{1}, T_{2}\right)$ ([10, Theorem 1.1).

\subsubsection{The map wind}

Let ${ }_{\mathcal{F}} \mathcal{U}=\left\{U \in \mathcal{U} \mid-1 \notin \operatorname{spec}_{e s s} U\right.$ ( i.e. $(U+1)$ is Fredholm) $\}$ The following "description" (definition) of wind is given ([10], Proposition 2.1):

Let $L: I=[0,1] \rightarrow_{\mathcal{F}} \mathcal{U}$ be a continuous path. There is a partition $0=t_{0},<t_{1}<\ldots<t_{n}=1$ of $[0,1]$, and positive real numbers $0<\epsilon_{j}<\pi, j=1, \ldots, n$, such that $\operatorname{ker}\left(f(t)-e^{i\left(\pi \pm \epsilon_{j}\right)}\right)=\{0\}$. Then wind $(f)$ is defined by

$$
\operatorname{wind}(f)=\sum_{j=1}^{n} k\left(t_{j}, \epsilon_{j}\right)-k\left(t_{j-1}, \epsilon_{j}\right)
$$

where

$$
k\left(t, \epsilon_{j}\right)=\sum_{0 \leq \theta<\epsilon_{j}} \operatorname{dim} \operatorname{ker}\left(f(t)-e^{i(\pi+\theta)}\right)
$$

This definition is independent of the choice of the partition of the interval and of the barriers $\epsilon_{j}$.

Definition of the spectral flow of $\mathbf{L}, s f(L)$ :

$$
s f(L)=s f(L, I)=\operatorname{wind}(\kappa \circ L)
$$




\subsubsection{Homotopy}

(Cf. J. Dieudonné [19]). Let $L_{1}$ and $L_{2}$ two paths defined in the same intervalle $I=[a, b]$, and $A$ an open set in $\mathbb{C}$, such that $L_{1}(I) \subset A$ and $L_{2}(I) \subset A$. An homotopy of $L_{1}$ to $L_{2}$, in $A$, is a continuous map $\varphi:(t, \xi) \in I \times[\alpha, \beta](\alpha<\beta \in$ $\mathbb{R}) \rightarrow A$ such that $\varphi(t, \alpha)=L_{1}(t)$ and $\varphi(t, \beta)=L_{2}(t)$ in $I$. Then the two paths $L_{1}$ and $L_{2}$ are said to be homotopes in A

The spectral flow defined by 322 is invariant under homotopies leaving the end points fixed ([10] Proposition 2.3).

\subsection{Spectral flow and bifurcation}

\subsubsection{Differentiable functions in Banach spaces}

Let $E$ and $F$ be two Banach spaces and $U$ a neighborhood of 0 in $E$. We say that the maps $f_{1}: U \rightarrow F$ and $f_{2}: U \rightarrow F$ are tangent at $a \in U$ if

$$
m(r)=\sup _{\|x-a\| \leq r}\left\|f_{1}(x)-f_{2}(x)\right\|
$$

satisfies the condition

$$
\lim _{r \rightarrow 0, r>0} \frac{m(r)}{r}=0 \text { or } \mathrm{m}(\mathrm{r})=\mathrm{o}(\mathrm{r})
$$

Definition 33. A map $f: U \rightarrow F$ is differentiable at the point $a \in U$ if there exists a $g \in \mathcal{L}(E, F)$ such that

$$
\|f(x)-f(a)-g(x-a)\|_{F}=o\left(\|x-a\|_{E}\right) \text { as } x \rightarrow a
$$

where $\mathcal{L}(E, F)$ is the space of linear continuous maps from $E$ to $F$ with its usual norm. The map $g$ is called the (Fréchet) derivative of $f$ at the point $a$ and is denoted by $f^{\prime}(a)$ or $D f(a)$. (Cf. H. Cartan [11] )

Derivative of second order (Cf. [19]. Let $f$ be a continuously differentiable function in an open set $A$ of a Banach space $E$ to a Banach space $\mathrm{F}$. Then $D f$ is a continuous map from A to $\mathcal{L}(E, F)$. If $D f$ is differentiable at the point $a \in A, f$ is said to be twice continuously diffferentiable at $a \in A$ and the derivative of $D f$ at the point $a$ is called the second derivative of $f$ at $a$, and is denoted by $f^{\prime \prime}(a)$ or $D^{2} f(a)$.

Partial derivatives Let $E_{1}, E_{2}, F$ be Banach spaces, $E=E_{1} \times E_{2}, A$ be an open set of $E$, and $f$ a differentialbe map from $A$ to $F$ and $a=\left(a_{1}, a_{2}\right) \in A$

Definition 34. The map $f$ is said differentiable, at the point $\left(a_{1}, a_{2}\right)$, with respect to the first [resp. second variable] if the partial map $x_{1} \rightarrow f\left(x_{1}, a_{2}\right)$ [resp. $x_{2} \rightarrow f\left(a_{1}, x_{2}\right)$ ] is differentiable in $a_{1}$ [resp. $a_{2}$ ]. These derivatives are called partial derivative with respect to the first variable [resp. the second variable] at the point $\left(a_{1}, a_{2}\right)$ and are denoted by $D_{x_{1}} f\left(a_{1}, a_{2}\right)\left(\in \mathcal{L}\left(E_{1}, F\right)\right)$ [resp. $D_{x_{2}} f\left(a_{1}, a_{2}\right)\left(\in \mathcal{L}\left(E_{2}, F\right)\right)$ ]. Moreover the gradient of $f$ at the point $\left(a_{1}, a_{2}\right)$ is defined by

$$
\nabla f\left(a_{1}, a_{2}\right)=D_{x_{1}} f\left(a_{1}, a_{2}\right) \times D_{x_{2}} f\left(a_{1}, a_{2}\right) \in \mathcal{L}\left(E_{1}, F\right) \times \mathcal{L}\left(E_{2}, F\right)
$$

Remark 30. The above definition can be extented, in the same way, when $E$ is a product of more than two spaces. 


\subsubsection{Bifurcation}

(Cf. 23]) Let $f(I \times U) \rightarrow X$ be a continuously differentiable mapping defined on the product of the interval $I=[a, b]$ with a neighborhood $U$ of the origin in a real Banach space $\mathrm{X}$, such that $f(\lambda, 0)=0$ for all $\lambda \in I$. Solutions of the equation $f(\lambda, x)=0$ of the form $(\lambda, 0)$, are called trivial. A bifurcation point for solutions of the equation $f(\lambda, x)=0$ is a point $\lambda^{*} \in I$ such that every neighborhood of $\left(\lambda^{*}, 0\right)$ contains nontrivial solutions of this equation. Let, for $\lambda \in I$, $L_{\lambda}=D_{x}(f(\lambda, x))(\lambda, 0) \in \mathcal{L}(X, X)$ be the derivative of $f(\lambda, x)$ with respect to $x$, at the trivial solution. By the implicit function theorem, bifurcation can occur only at points where $L_{\lambda}$ is singular i.e. is noninvertible.

The following result is presented in P. M. Fitzpatrick et al. [24] Theorem A. Let $I=[a, b]$ be an interval of real numbers, $X$ be a real separable Hilbert space and $U$ be a neighborhood of $I \times\{0\}$ in $\mathbb{R} \times X$ on which the $C^{2}$ function $\psi:(\lambda, x) \in U \rightarrow \psi(\lambda, x) \in \mathbb{R}$ has the property that, for each $\lambda \in I, 0$ is a critical point of $\psi_{\lambda} \equiv \psi(\lambda, \cdot)$. It is assumed that the Hessian $L_{\lambda}$ of $\psi_{\lambda}$, at 0 , is Fredholm, and that $L_{a}$ and $L_{b}$ are nonsingular. Then, if the spectral flow of $\left\{L_{\lambda}\right\}$ on the interval $I$ is nonzero, every neigborhood of $I \times\{0\}$ contains points of the form $(\lambda, x)$ where $x \neq 0$ is a critical point of $\psi_{\lambda}$.

\subsubsection{Examples}

Example 14. ([24])

Let $I=[a, b]$ and $H=(\lambda, t, u) \in I \times \mathbb{R} \times \mathbb{R}^{2 n} \rightarrow H(\lambda, t, u) \in \mathbb{R}$ be a twice continuously differentiable function, $2 \pi$-periodic in $t$ with $H(\lambda, t, 0) \equiv 0$. The following Hamiltonian system for the differentiable function $u: \mathbb{R} \rightarrow \mathbb{R}^{2 n}$, is considered:

$$
J u^{\prime}(t)+\nabla_{u} H(\lambda, t, u(t))=0, \quad u(0)=u(2 \pi)
$$

where

$$
J=\left(\begin{array}{cc}
0 & -\mathrm{Id}_{n} \\
\operatorname{Id}_{n} & 0
\end{array}\right)
$$

is the symplectic $2 n \times 2 n$ matrix. The authors make assumptions under which they can apply their previous results [23] and show that bifurcation of $2 \pi$-periodic orbits from the branch of equilibrium arises. Here

$$
X=H^{\frac{1}{2}} \equiv H^{\frac{1}{2}}\left(S^{1}, \mathbb{R}^{2 n}\right),
$$

the function $\psi: I \times H^{\frac{1}{2}} \rightarrow \mathbb{R}$, is defined by

$$
\psi(\lambda, u)=\frac{1}{2} \Gamma(u, u)+\int_{0}^{2 \pi} H(\lambda, t, u(t)) d t
$$

where $\Gamma(u, v)=\int_{0}^{2 \pi}\left\langle J u^{\prime}(t), v(t)\right\rangle d t, \forall v \in H^{\frac{1}{2}}$. The Hessian $L_{\lambda}$ of $\psi(\lambda, \cdot)$ defined by $L_{\lambda}=D_{u} F(\lambda, 0)$, where

$$
F(\lambda, u)=\nabla_{u} \psi(\lambda, u), \quad(\lambda, u) \in I \times H^{\frac{1}{2}}
$$

is Fredholm. The spectral flow $s f(L, I)$ of the path $L=\left\{L_{\lambda}\right\}_{\lambda \in I}$ is nonzero.

Example 15. (Cf. N. Waterstraat [61]).

Let $\Omega$ be a bounded domain in $\mathbb{R}^{N}, N \in \mathbb{N}$, with a smooth boundary $\partial \Omega, I=[0,1]$. Let $a, b, c: I \times \bar{\Omega} \rightarrow \mathbb{R}$ and $G: I \times \bar{\Omega} \times \mathbb{R}^{2} \rightarrow \mathbb{R}$ be $C^{2}$-functions. Systems of elliptic partial differential equations of the form

$$
\left\{\begin{array}{l}
-\Delta u=b_{\lambda}(x) u+c_{\lambda}(x) v+G_{v}^{\prime}(\lambda, x, u, v) \text { in } \Omega \\
-\Delta v=a_{\lambda}(x) u+b_{\lambda}(x) v+G_{u}^{\prime}(\lambda, x, u, v) \text { in } \Omega \\
u=v=0 \text { on } \partial \Omega
\end{array}\right.
$$


are considered. Here $G_{u}^{\prime}(\lambda, x, 0,0)$ and $G_{v}^{\prime}(\lambda, x, 0,0)$ are assumed to be 0 for all $(\lambda, x) \in I \times \Omega$. Aditionnal assumptions are made on $G$ and its derivatives such that the following results are justified. Let $D^{2} G(\lambda, x, u, v)$ denote the Hessian matrix of $G(\lambda, x, \cdot, \cdot): \mathbb{R}^{2} \rightarrow \mathbb{R}$, at the point $(u, v) \in \mathbb{R}^{2}$ with $D^{2} G(\lambda, x, 0,0)=0$. Let $E$ be the Hilbert space $H_{0}^{1}(\Omega, \mathbb{R}) \times H_{0}^{1}(\Omega, \mathbb{R})$ with the corresponding scalar product $\langle\cdot, \cdot\rangle_{E}$, and for $z=(u, v) \in E$, define the map

$$
f_{\lambda}(z)=\int_{\Omega}\langle\nabla u, \nabla v\rangle d x-\frac{1}{2} \int_{\Omega}\left(a_{\lambda}(x) u^{2}+2 b_{\lambda}(x) u v+c_{\lambda}(x) v^{2}\right) d x-\int_{\Omega} G(\lambda, x, u, v) d x
$$

which is $C^{2}$. Moreover, $f_{\lambda}(0)=0$ for all $\lambda \in I$. For $z=(u, v) \in E$, the map $\tilde{z}=(\tilde{u}, \tilde{v}) \in E$ to

$$
D_{0}^{2} f_{\lambda}(z, \tilde{z})=\int_{\Omega}\langle\nabla u, \nabla \tilde{v}\rangle d x+\int_{\Omega}\langle\nabla \tilde{u}, \nabla v\rangle d x-\int_{\Omega}\left(a_{\lambda}(x) u \tilde{u}+b_{\lambda}(x)(\tilde{u} v+u \tilde{v})+c_{\lambda}(x) v \tilde{v}\right) d x
$$

is a continuous linear form on E. From the Riesz representation theorem, there exists $L_{\lambda}(z) \in E$ such that

$$
\left\langle L_{\lambda} z, \tilde{z}\right\rangle_{E}=D_{0}^{2}\left(f_{\lambda}(z, \tilde{z})\right) \quad z, \tilde{z} \in E
$$

and $L_{\lambda} \in \Phi_{S}(E)$. Therefore, the path $L=\left\{L_{\lambda} \mid \lambda \in I\right\}$ is a path of bounded linear self-adjoint, Fredholm operators and the spectral flow $s f(L, I)$ is well defined. It is proved that if the linearized systems

$$
\left\{\begin{array}{l}
-\Delta u=b_{\lambda}(x) u+c_{\lambda}(x) v \text { in } \Omega \\
-\Delta v=a_{\lambda}(x) u+b_{\lambda}(x) v \text { in } \Omega \\
u=v=0 \text { on } \partial \Omega
\end{array}\right.
$$

have no nontrivial solution for $\lambda=0$ and $\lambda=1$, then the spectral flow $s f(L, I)$, estimated in terms of the coefficients of (336), is nonero. This implies the existence of a bifurcation point $\lambda^{*} \in(0,1)$ for the family of equations (332) ( 61 , Theorems 2.1 and 4.2 ).

\section{Conflict of Interests}

Th author declares that there is no conflict of interests regarding the publication of this paper.

\section{References}

[1] D.R. Adhikari, E. Stachura 2020. General p-curl systems and duality mappings on Sobolev spaces for maxwell equations, Electronic Journal of Differential equations, 116, 1-22. 397

[2] G. Allaire 2002. Shape optimization by the homogenization method. Applied Mathematical Sciences 146, Springerverlag. $392,393,394$

[3] W. Ao, J. Wei, M. Winter 2020. Stable pikes clusters on a compact two-dimensional Riemannian manifold, Journal of Differential Equations,268, 3665-3704. 383

[4] V. Arnold 1965. Small denominators, 1: Mappings of the circumference onto itself. AMS Translations, 46, 213-288. (Russian original published in 1961). 390

[5] V. Arnold 1983. Geometrical methods in the Theory of Ordinary Differential Equations. Springer-Verlag · 390

[6] J. Arazy and L. Zelenko 2006. Virtual eigenvalues of high order Schrödinger operator, Integral Equations and Operator Theory, 55, 189-231 and 305-345. 404 
[7] N. Aronszajn 1950. Theory of reproducing kernels, Transactions of the American Mathematical Society 68, 337-404. 388

[8] G. Auchmuty 2017. The S. V. D. of Poisson Kernel, Journal of Fourier Analysis and Applications, 23, 1517-1536. 385, 386, 387

[9] S. Axler, P. Bourdon, W. Ramey 2001. Harmonic Function Theory, Graduate Texts in Mathematics, Springer, Second Edition. 387

[10] B. Booss-Bavnbek, M. Lesh, J. Phillips 2005. Unbounded Fredholm operators and spectral flow, Canadian Journal of Mathemtics, 57 no.2, 225-250. 411, 412 , 413

[11] H. Cartan 1971. Calcul Differentiel, Hermann, Collection Méthodes, Paris, 1967413

[12] A. Chechkina, C. D’Apice, U. De Maio 2019. Rate of convergence of eigenvalues to singularly perturbed Steklov-type problem of elasticity system, Applicable Analysis, 98 no. 1-2, 32-44. 396, 397

[13] E.A. Coddington and N. Levinson 1955. Theory of differential equations, McAGraw-Hill Book Company, New-York $(1955)$. 388,389

[14] H. O. Cordes, J. P. Labrousse 1963. The invariance of the index in the metric space of closed operators, Journal of Mathematics and mechanics, 12 No. 5, 693-719. 403

[15] E.B. Davies 1999. Pseudo-spectra, the harmonic oscillator and complexes resonances. Royal Society of London Proceedings :Mathematical, Physical and Engineering Sciences, 455 no. 1982, 585-599. 406

[16] E. B. Davies 2002. Non-self-adjoint differential operators.Bulletin of The London Mathmatical Society,34, 513-532. 408

[17] E.B. Davies 2007. Linear operators and their spectra. Cambridge Studies in Advanced Mathematics ,106. 406

[18] A. Denjoy 1932. Sur les courbes définies par les équations différentielles à la surface du tore, Journal de Mathématiqus pures et Appliquées, 11, série 9, 333-375. 392

[19] J. Dieudonné, 1968. Eléments d'analyse. Tome I: Fondements de l'Anlyse Moderne, Cahiers Scientifiques, Fasc.XXVIII, Gauthiers-Villars, Paris, xxi +390 pages. 403,413

[20] A. Doelman 2019. Pattern formation in reaction-diffusion systems- an explicit approach, Peletier Complexity science, World Scientific Publishing Reviews, Hackensack N. J, 129-182. 374, 375, 376

[21] G. Domokos and P. Holmes 2003. On nonlinear boundary-value problems: ghosts, parasites and discretizations. The Royal Society of London. Proceedings. Series A. Mathematical, Physical, and Engineering Sciences, 459 no. 2034, 1535-1561. 405

[22] W. Feller 1950. An introduction to probability theory and its applications. Vol. I. John Wiley and Sons. 408

[23] M. Fitzpatrick, J. Pejsachowicz, L. Recht 1999. Spectral flow and bifurcation of critical points of strongly-indefinite functionals, Part I. General Theory. Journal of Functional Analysis, 162 , 52-95. 411, 414

[24] M. Fitzpatrick, J. Pejsachowicz, L. Recht 2000. Spectral flow and bifurcation of critical points of strongly-indefinite functionals, Part II. Bifurcation of Periodic Orbits of Hamiltonian Systems. Journal of Differential Equations, 163, $18-40414$ 
[25] [K. Furutani, N. Otsuki, 1991. Spectral flow and Maslov index arising from Lagrangian intersection. Tokyo Journal of Mathematics, 14, 135-150. 411.

[26] F. Gazzola, H-G. Grunau and G. Sweers 2010. Polyharmonic boundary value problems, Springer, Berlin. 383 . 384

[27] A. Gierer, H. Meinhardt 1972. A theory of biological pattern formation, Kibernetik, 12, 30-39. 381]

[28] I. C. Gohberg, M. G. Krein 1957. Fundamental aspects of defect numbers, root numbers and indexes of linear operators. Uspekhi Matematicheskikh Nauk, 2,3 (19), 60-107. 403

[29] G.H. Hardy and E.M. Wright 1990. An introduction to the theory of numbers, Fifth Edition, Oxford Science Publications. 391

[30] M.R. Herman 1979. Sur la conjugaison différentielle des difféomorphismes du cercle à des rotations. Publications Mathématiques de l'Institut des Hautes Etudes Scientifiques, 49, 5-234. 392

[31] M.R. Herman 1985. Simple proofs of local conjugacy theorems for diffeomorphisms of the circle with almost every rotation number, Boletim da Sociedade Brasileira de Matemática, 16 no.1, 45-83 392

[32] R. Henry, D.Krejčiřík 2017. Pseudospectra of the Schrödinger operator with discontinuous complex potential, Journal of Spectral Theory, 7: 659-697. 404, 406

[33] D. Huet 2017. A survey of topics related to partial differential equations, Editions Universitaires Européennes, Omniscriptum Marketing DEU GmbH, Saarbrüken. 404

[34] D. Huet 2019, A survey of topics related to functional analysis and operator theory. 2019. hal-01952296v2. 374

[35] D. Huet 2020, A survey of differential operators related to applied sciences. 2020. hal-02940081. 374

[36] T. Kato 1966. Perturbation theory for linear operator, Springer-Verlag New-York Inc. 402,403

[37] C.A. Klausmeier 1999. Regular and irregular patterns in semi-arid vegetation, Science 284,1826-1828. 378

[38] D. Krejčiřík, P. Siegl, M. Tater, J. Viola 2015. Pseudospectra in non-Hermitian quantum mechanics, Journal of Mathematical Physics 56, 103513. 408,409

[39] W. Kühnel 2015. Differential Geometry. Student Mathematical Library, 77. American Mathematical Society, xii +402 pp. 381

[40] W. de Melo and S. van Strien 2018. One dimensional dynamics, A series of Modern Surveys in Mathematics. Springer-Verlag. 392

[41] P. Monk 2003. Finite element method for Maxwell's equations, Clarendon Press, Oxford. $398,399,400$

[42] David S. Morgan, A. Doelman, Tasso J. Kaper 2000. Stationary periodic patterns in the Gray-Scott model, Methods and Applications of Analysis, 7 no. 1, 105-150. 376, 377, 379

[43] V.V. Nemytskii and V.V. Stepanov 1960. Qualitative theory of differential equations, Princeton Universirty Press, Princeton 1960. 389

[44] O.A. Oleinik, A.S. Shamaev, G.A. Yosifian 1992. Mathematical problems in elasticity and homogenization, Studies in Mathematics and its Applications, North-Holland. 393, 394, 395, 396 
[45] H. Poincaré 1881-1886. Mémoire sur les courbes définies par une équation différentielle, Journal de Mathématiques Pures et Appliquées, 18817 375-422, 18828 251-286, 18851 167-244, 18862 151-217. 392

[46] L. Prigozhin 1996. On the Bean critical state model in superconductivity. European Journal of applied athematics, 7, 237-247. 400

[47] L. Prigozhin 1996. The Bean model in superconductivity: variational formulation and numerical solution, Journal of Computational Physics, 129, 190-200. 400, 401

[48] S.C. Reddy, L. N. Trefethen 1994. Pseudospectra of the Convection-Diffusion Operator, SIAM Journal on Applied Mathematics, 54 no 6 ,1634-1649. 409, 410, 411

[49] M. Reed and B. Simon 1972. Methods of modern mathematical physics, volume 1: Functional analysis, Academic Press, New-York. 387

[50] M. Schechter 1981. Operator Method in Quantum mechanics. Elsevier, North-Holland. 407, 408

[51] L. Schwartz,1970. Analyse, Topologie Générale et Analyse Fonctionnelle, Hermann, Collection Enseignement des Sciences No. 11, Paris, 433 pp. 402

[52] P. Sieglej D. Krvciř́k 2012. On the metric operator for the imaginary cubic operator, Physical Review D, 86, $121702(\mathrm{R}) .409$

[53] SMF 2018. La Gazette des mathématiciens Avril 2018: Jean-Christophe Yoccoz, Numero special, Societé Mathématique de France. 390

[54] E.C. Titchmarsh 1988. The Theory of Functions, second edition, Oxford University Press. 404

[55] . T.L. Trefethen 1997. Pseudospectra of linear operators. Siam Review, 39 no. 3, 383-406. 410

[56] T.L. Trefethen and M. Embree 2005. Spectra and Pseudospectra, Princeton University Press, Princeton, NJ. 402 $403,404,405$

[57] W. Tse, J.C. Wei, M. Winter 2010. The Gierer-Meinhardt system on a compact two-dimensional Riemannian manifold: Interaction of gaussian curvature and Green's function, Journal of Mathématiqus pures et appliquées, 94, 366-397. 381,382

[58] A.M. Turing 1990. The chemical basis of morphogenesis, Bulletin of Mathematical Biology, 52 no.1-2, 153-197. 374

[59] S. van der Stelt, A. Doelman, G.M. Hek, J.D.M. Rademacher 2013. Rise and fall of periodic paterns for a generalized Klausmeier-Gray-Scott model, Journal of Nonlinear Sciences, 23 no.1, 39-95. 371, 378, 379, 380, 381,

[60] C. E. Waine 2008. An introduction to KAM theory. Preprint. 390

[61] N. Waterstraat 2018. Spectral flow and bifurcation for a class of strongly indefinite elliptic systems. Proceedings of the Royal Society of Edinburgh, 148A, 1097-1113. 414, 415

[62] L. Wen 2016. Differentiable Dynamical Systems. An introduction to structural stability and hyperbolicity, Graduate Studies in Mathematics, 173, American Mathematical Society, Providence, Rhode Island. 389

[63] J-C. Yoccoz 2002. Analytic linearization of circle diffeomorphism. Dynamical systems and small divisors, Springer: 125-173. 390,392

[64] H. M. Yin, B. Q. Li, J. Zou 2002. A degenerate evolution system modeling Bean's critical-state type-II superconductors, Discrete and continuous dynamical systems, 8 no.3, 781-794. 397, 401 